\title{
Biomimeting ultra-small lipid nanoconstructs for glioblastoma treatment: A computationally guided experimental approach
}

\author{
Maria Mendes $^{\mathrm{a}, \mathrm{b}, \mathrm{c}, 1}$, João Basso ${ }^{\mathrm{a}, \mathrm{b}, \mathrm{c}, 1}$, Jessica Silva ${ }^{\mathrm{a}, \mathrm{c}, 1}$, Tânia Cova ${ }^{\mathrm{b}}$, João Sousa ${ }^{\mathrm{a}, \mathrm{b}}$, Alberto Pais ${ }^{\mathrm{b}}$, \\ Carla Vitorino ${ }^{\mathrm{a}, \mathrm{b}, \mathrm{c}, *}$ \\ ${ }^{a}$ Faculty of Pharmacy, University of Coimbra, Pólo das Ciências da Saúde, Azinhaga de Santa Comba, 3000-548 Coimbra, Portugal \\ ${ }^{\mathrm{b}}$ Coimbra Chemistry Centre, Department of Chemistry, University of Coimbra, Rua Larga, 3004-535 Coimbra, Portugal \\ ${ }^{\mathrm{c}}$ Centre for Neurosciences and Cell Biology (CNC), University of Coimbra, Faculty of Medicine, Rua Larga, Pólo I, 1st Floor, 3004-504 Coimbra, Portugal
}

\section{A R T I C L E I N F O}

\section{Keywords:}

Drug repurposing

Glioblastoma

Ultra-small nanostructured lipid carriers

Biomimeting

Design of experiments

Process parameters

\begin{abstract}
A B S T R A C T
Ultra-small nanostructured lipid carriers (usNLCs) are stable, biocompatible and biodegradable colloidal systems, claiming a broad set of advanced features suitable for cancer drug delivery. To unleash their potential in glioblastoma research and therapy, we have developed an usNLC prototype able to co-encapsulate atorvastatin calcium and curcumin, as repurposed drugs previously screened from molecular dynamics simulations. The novelty not only relies on the drug repositioning approach, but also on a robust computational methodology utilized for formulation optimization, under the umbrella of multivariate analysis and full factorial designs. A coating procedure with red blood cell membranes is ultimately hypothesized, aiming at integrating the biomimetic concept into usNLCs for glioblastoma therapeutics.

The formulation composition and process parameters, that demonstrated a high-risk level for the final quality and performance of the usNLCs, include the solid:liquid lipid ratio, type and concentration of liquid lipids and surfactants, along with the type of production method. Particles with an average diameter of ca. $50 \mathrm{~nm}$, and a polydispersity index lower than 0.3 were produced, exhibiting high stability, up-scalability, drug protection and sustained co-release properties, meeting the suitable critical quality attributes for intravenous administration. Also, a Taguchi design was successfully applied to optimizing usNLCs as cell membrane-coating technology.
\end{abstract}

\section{Introduction}

Nanotechnology has been identified as a potential therapeutic strategy in the field of oncology, including the treatment of glioblastoma, the most frequent, aggressive and deadly type of brain cancer (Karim et al., 2016; Pourgholi et al., 2016; Urbańska et al., 2014). The current standard of care for glioblastoma relies on surgery, followed by radiotherapy and temozolomide (TMZ) chemotherapy (Stupp et al., 2005). Notwithstanding, the median survival rate usually does not extend beyond 15 months in patients diagnosed with primary glioblastoma (Alifieris and Trafalis, 2015). The poor prognosis reported is a consequence of its intrinsic characteristics, including location, cellular heterogeneity, diffuse infiltrative behavior on surrounding brain structures, and chemotherapy resistance (Ohgaki and Kleihues, 2005; Lee, 2016).

How can we improve glioblastoma healthcare? Finding out renewed chemical entities stands out as a key strategy. In addition to TMZ, other non-cytotoxic drugs have been evaluated as potential chemotherapeutic

Abbreviations: A, atorvastatin; ANOVA, One-way analysis of variance; ATO, atorvastatin calcium; ATB, Automated Topology Builder; BBB, blood-brain barrier;

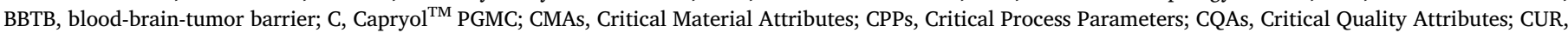

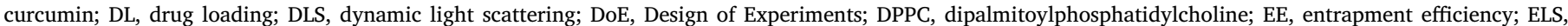

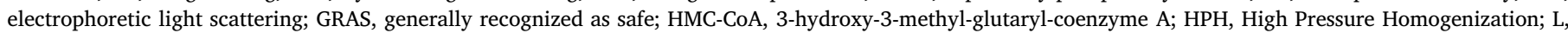

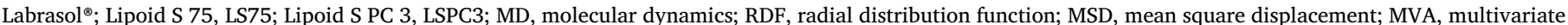

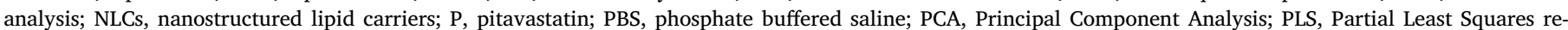

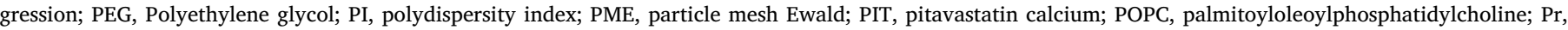

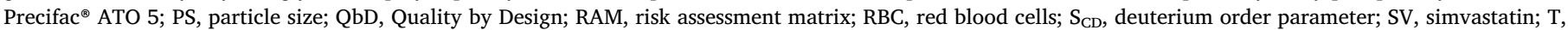
Transcutol ${ }^{\circledR}$ HP; T80, Tween ${ }^{\circledR}$ 80; TMZ, temozolomide; US, Ultrasonication; usNLCs, ultra-small nanostructured lipid carriers; ZP, zeta potential.

* Corresponding author at: Faculty of Pharmacy, University of Coimbra, Pólo das Ciências da Saúde, Azinhaga de Santa Comba, 3000-548 Coimbra, Portugal.

E-mail address: csvitorino@ff.uc.pt (C. Vitorino).

${ }^{1}$ These authors contributed equally to this work and should be regarded as co-first authors. 
agents to be repositioned in glioblastoma treatment, covering several pharmacotherapeutic classes (Basso et al., 2018). Among them, curcumin (CUR) and different statins have demonstrated encouraging results. CUR, or diferuloymethane, is a hydrophobic agent derived from the plant Curcuma longa, displaying anti-inflammatory, anti-oxidant and anticancer properties. Interestingly, it has been reported its potential benefits as a therapeutic agent for the treatment of glioblastoma through several mechanisms of action. However, CUR exhibits low water solubility and oral bioavailability, thus affecting its therapeutic efficacy (Sordillo et al., 2015; Zhuang et al., 2012; Luthra and Lal, 2016; Gersey et al., 2017). Statins, including atorvastatin calcium (ATO), are 3-hydroxy-3-methyl-glutaryl-coenzyme A (HMG-CoA) reductase inhibitors, with wide applicability in dyslipidemia treatment. By blocking the synthesis of mevalonate, a rate limiting step of the biosynthesis of cholesterol, they have also demonstrated potential cytotoxic activity over several cancer types, including glioblastoma. Similarly to CUR, ATO also possesses a lipophilic behavior, low solubility and low oral bioavailability (Vasilev et al., 2018; Jones et al., 2017; Prabhu and Patravale, 2016).

Other approaches are directed to formulation aspects (Carlsson et al., 2014). Lipid nanoparticles, including nanostructured lipid carriers (NLCs), have been broadly explored as promising brain targeted delivery vehicles in order to overcome such limitations of the conventional glioblastoma treatment (Mendes et al., 2018; Vitorino et al., 2013). NLCs are colloidal carriers with a solid matrix at both room and body temperatures, composed of spatially incompatible solid and liquid lipids in appropriate proportions, displaying a mean particle size in the nanometer range. More recently, ultra-small NLCs (usNLCs) have sparked particular interest, stemming from their size typically below $100 \mathrm{~nm}$, obtained by employing higher ratios of liquid to solid lipids. Consequently, they exhibit several advantages as pharmaceutical carriers comparing to the other lipid-based nanoparticles, such as high physicochemical stability and drug loading, good biocompatibility and biodegradability, use of generally recognized as safe (GRAS) excipients, easiness towards large-scale production, surface functionalization, controlled release and drug protection, and versatile entrapment of both hydrophobic and hydrophilic drugs (Khan et al., 2015; Tapeinos et al., 2017; Singh and Lillard, 2009).

But, how can we design usNLCs in a more efficient way? usNLCs composition and production method comprise a set of factors, designed as critical material attributes (CMAs) and critical process parameters (CPPs), respectively, that influence the critical quality attributes (CQAs) and, consequently, the quality and performance of the final product. Thus, the optimization step is fundamental in the development of these systems. Design of experiments (DoE) and Multivariate statistical analysis (MVA) methods have been proposed as a preliminary screening step to identify and evaluate the effect of such factors on CQAs and, subsequently, to select the optimal formulation (Mendes et al., 2018; Silva et al., 2018). It has been reported that in vivo biodistribution and the ability of intravenously administered nanoparticles to cross the blood-brain barrier (BBB) and blood-brain-tumor barrier (BBTB) are strongly affected by their size and surface properties (Miranda et al., 2017; Miranda et al., 2017).

The present work aims at designing and optimizing usNLCs for the treatment of glioblastoma considering their intravenous administration. Such procedures were strongly supported on a robust computational basis directed both to screen drugs and the formulation prototype (Fig. 1). First, molecular dynamics (MD) studies were carried out to select the drugs that best mimic the TMZ behavior among those repurposed as glioblastoma therapeutic agents for subsequent co-encapsulation into usNLCs. Subsequently, preliminary risk assessments were carried out so as to identify the most quality impacting variables (CQAs identification). Two multivariate analysis methods, namely principal component analysis (PCA) and partial least squares regression (PLS), and two full factorial designs were then applied as screening methods to assess the influence of the previously selected factors on the
CQAs. Specifically, the solid:liquid lipid ratio, type and concentration of liquid lipid and oily surfactant, the concentration of aqueous surfactant and the type of production method were varied in order to study their impact on particle size (PS), polydispersity index (PI), zeta potential (ZP), entrapment efficiency (EE), drug loading (DL), long-term stability and drug release. Finally, the design of a potential formulation involving the usNLC prototype cloaked with a red blood cell (RBC) membrane is presented as a biomimetic strategy to improve their functional performance, supported on a Taguchi design approach.

\section{Materials and methods}

\subsection{Materials}

Atorvastatin calcium was kindly provided by the Tecnimede Group (Sintra, Portugal). Curcumin (97\% purity) was purchased from Jinlan Pharm-Drugs Technology Co., Limited (China). Tween ${ }^{\circledR} 80$ (polysorbate 80) was acquired from Sigma-Aldrich (USA). Precifac ${ }^{\circledR}$ ATO 5 (cetyl palmitate), Transcutol ${ }^{\circledast}$ HP (diethylene glycol monoethyl ether), Labrasol $^{\circledR}$ (caprylocaproyl polyoxyl-8 glycerides) and Capryol ${ }^{\mathrm{TM}}$ PGMC (propylene glycol monocaprylate - type I) were kindly donated by Gattefossé (France). Lipoid S 75 (soy phospholipid) and Lipoid S PC 3 (hydrogenated soy phosphatidylcholine) were provided by Lipoid GmbH (Germany), while polyethylene glycol (PEG) 400 was acquired from Fluka $^{\mathrm{TM}}$. Water was purified (Millipore ${ }^{\circledR}$ ) and filtered through a $0.22 \mu \mathrm{m}$ nylon filter before use. All other reagents and solvents were of analytical or HPLC grade.

\subsection{Molecular dynamics studies}

\subsubsection{System components and simulation details}

MD simulations were performed for investigating the effect of some promising therapeutic agents in the treatment of glioblastoma, by inspecting the respective behavior into different lipid bilayers. TMZ, the current standard treatment in glioblastoma, displays good BBB penetration and is used as a reference compound in systems containing single solvated TMZ and a combination of TMZ and CUR (Yin et al., 2014). In an attempt to repurpose "old drugs", a set of different statin generations, including atorvastatin (A), atorvastatin calcium (ATO), pitavastatin (P), pitavastatin calcium (PIT) and simvastatin (SV), were further screened and compared with TMZ. The behavior of the statin molecule exhibiting the best profile to cross the model membranes was also inspected when combined with CUR. The latter was selected based on its lipophilic nature and the reported mechanisms of action in glioblastoma (Basso et al., 2018). Two different models of lipid membranes, dipalmitoylphosphatidylcholine (DPPC) and palmitoyloleoylphosphatidylcholine (POPC), were used to mimic the BBB and glioblastoma cell membranes in systems containing TMZ and CUR. DPPC and POPC are able to serve as a model for the BBB due to the presence of phosphatidylcholine, the major lipid type within cellular membranes (Shamloo et al., 2016; Miranda et al., 2018). The lipid bilayer which better describes the potential diffusion behavior through the BBB was selected for the remaining simulations of the study. Specifically, each system contains a fully hydrated bilayer, consisting of 128 lipid molecules equally distributed by two leaflets. The topology for lipid membranes (DPPC/POPC) was obtained from the website of the Biocomputing Group at University of Calgary (29). The topology for each compound (TMZ, CUR and statins) was generated using the Automated Topology Builder (ATB) platform (Malde et al., 2011). All simulations were carried out under GROMACS (version 4.5.6) (Hess et al., 2008) using simple point charge (SPC) water and the united-atom lipid parameters of Berger and co-workers (Berger et al., 1997). These parameters were combined with a GROMOS (53a6) representation of the TMZ, CUR and statins (Leong, 2016; Piggot et al., 2012).

The systems containing a membrane bilayer were built for single compounds (TMZ, CUR and each statin) and for different combinations 


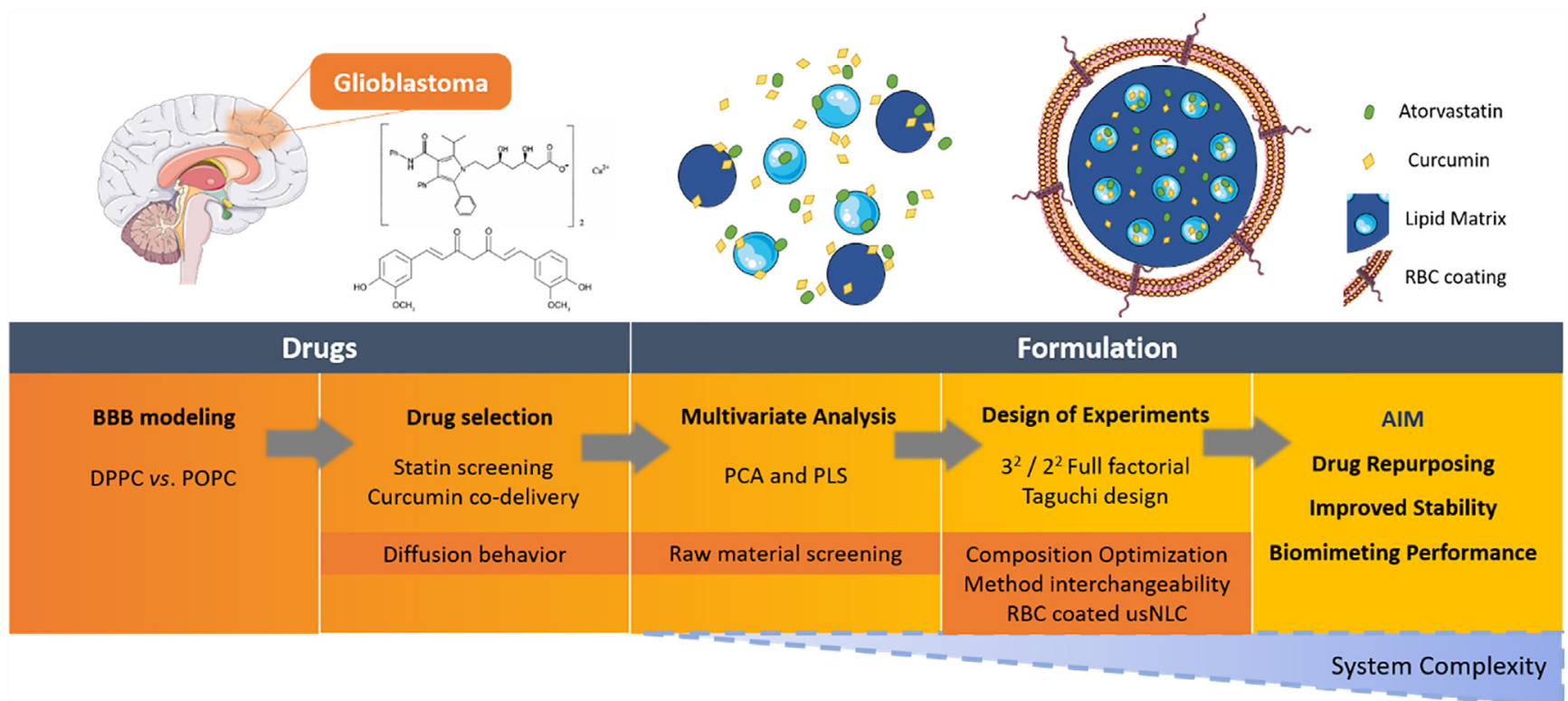

Fig. 1. Workflow proposed for the development of biomimeting uNLCs.

between them. One or two statin molecules were incorporated in the bilayers, for the non-ionic and ionic forms, respectively. MD simulations were performed for a total of seventeen systems. The interaction of each compound with the surrounding lipids, the formation of small aggregates/shells (e.g. between TMZ and CUR molecules), as well as the preferential positioning of the compounds on the lipid bilayer, were evaluated. All MD simulations were performed in the NPT ensemble under periodic boundary conditions. A standard time step of 2 fs was applied for both the equilibration and production runs. Non-bonded interactions were computed on the basis of a neighbor list, updated every 10 ps. For Lennard-Jones interactions, a cut-off at 1-4 nm was applied, while the long-range electrostatic interactions were treated with the particle mesh Ewald (PME) method. The V-rescale and Berendsen external baths were used to keep the temperature and pressure at $325 \mathrm{~K}$ and 1 bar throughout the simulation, respectively. Each system was firstly subjected to an energy minimization step. All systems were then left to evolve up to $85 \mathrm{~ns}$, using the LINCS algorithm to constrain bond lengths (Hess et al., 1997). The first $25 \mathrm{~ns}$ of the simulation time were used for equilibration, while the last 60 ns were used for the analyses, including density profiles, mean square displacement (MSD), order parameter, and radial distribution functions (RDF). Representative configurations of each system were extracted using the VMD 1.9 software (Humphrey et al., 1996).

\subsection{Preliminary risk assessment}

Risk assessment aims to identify, analyze and evaluate risks associated with the development of a given formulation, including usNLCs (ICH, 2006). An overall risk assessment, embodied by an Ishikawa diagram, was carried out in order to define a set of potential cause-andeffect relationships among the critical factors, namely critical material attributes (CMAs) and critical process parameters (CPPs), that affect the desired quality of the final product, represented by their critical quality attributes (CQAs) for intravenous administration. Additionally, a risk assessment matrix (RAM) was performed by prioritizing high risk factor (s). This matrix displays the qualitative potential risk(s), ranked as low-, medium- or high-risk, related to each of the CMAs and CPPs on the potential CQAs of the usNLCs.

\subsection{Preparation of nanocarriers}

The preparation of NLCs was carried out according to the method described elsewhere (Mendes et al., 2018). Briefly, 7.5\% (w/w) of lipid phase, comprising solid lipid and liquid lipids in an appropriate ratio, the oily phase surfactant, ATO $(5 \%, w / w)$ and CUR $(2.5 \%, w / w)$ were blended and molten at $80{ }^{\circ} \mathrm{C}$. Subsequently, $30 \mathrm{~mL}$ of aqueous phase, consisting in a solution of the surfactant Tween ${ }^{\circledast} 80$, was heated to the same temperature. Lipid and aqueous phases were pre-emulsified using a high-speed stirrer (Ultra-Turrax X 10/25; Ystral GmbH, Dottingen, Germany) at $24000 \mathrm{rpm}$ for $1 \mathrm{~min}$, followed by ultrasonication (US, Branson ${ }^{\circledast}$ Sonifier 250; Branson Ultrasonics Corporation, Connecticut, USA) with an amplitude frequency of $100 \%$ during $2.5 \mathrm{~min}$ or, alternatively, by high pressure homogenization (HPH, EmulsiFlex-C3; Avestin, Inc., Ottawa, Canada) at a pressure of 1000 bar for $2.5 \mathrm{~min}$. The resulting dispersion was then stored at $4{ }^{\circ} \mathrm{C}$ to form the NLCs. Note that to optimize the most promising NLC, in particular to obtain usNLCs, the solid:liquid lipid ratio, the type and concentration of liquid lipid and oily phase surfactant, the concentration of aqueous surfactant Tween ${ }^{\circledR} 80$, as well as the type of production method, were modified according to the defined planning variables. The term NLCs will be crosswise used in the following sections, to refer the multitude of lipid nanoparticle formulations, including usNLCs, obtained throughout all the development steps.

\subsection{Nanocarrier optimization}

The optimization of the composition and the processing parameters of usNLCs is a very important step in the development of this type of systems to obtain a final product with the desired CQAs (Silva et al., 2018). The optimization strategy was performed using R 3.1.1 and RStudio, by applying principal component analysis (PCA) and partial least square regression (PLS), as a first preliminary screening. Secondly, considering the DoE methodology, the three-level, $3^{\mathrm{k}}$, and two-level, $2^{\mathrm{k}}$, full factorial designs were performed to construct polynomial models for the optimization of the usNLCs, in what concerns the prototype construction and the production method interchangeability. Such designs have the peculiarity of requiring relatively few runs with high number of variables. 


\subsubsection{Multivariate data analysis}

PCA and PLS were applied after standard normalization to evaluate the quantitative and qualitative effects of the type and amount of liquid lipids and oily surfactant on PS, PI and ZP. The main goals of PCA are to reduce the dimensionality of the data set composed of different NLC formulations in terms of the most discriminating variables (Abdi and Williams, 2010; Jolliffe, 2002; Mutihac and Mutihac, 2008), while PLS allows finding the optimal relations between the measurable responses (dependent variables) and the set of predictors (independent variables) (Mutihac and Mutihac, 2008; Abdi, 2010). PLS models were calculated using the kernel algorithm and cross-validation (see references (Silva et al., 2018; Varmuza and Filzmoser, 2009) for details). Both methods require a spatial description of each lipid nanoparticle formulation as points in Euclidian space, described by several physicochemical attributes.

\subsubsection{Design of experiments}

$3^{\mathrm{k}}$ full factorial design

The first design was performed to provide the empirical mathematical models able to describe the effects of the amount of aqueous surfactant and the composition of lipid phase (independent variables) on PS, PI, ZP, EE and DL (dependent variables). Different concentrations of the aqueous surfactant Tween ${ }^{\circledR} 80$ (factor 1 ) were considered, namely 1,3 and $5 \%(\mathrm{w} / \mathrm{w})$, represented by the coded levels $-1,0$ and +1 , respectively. Also, the composition of lipid phase (factor 2) was analyzed considering three solid:liquid lipid ratios, 25:75, 50:50 and $75: 25$, corresponding to $-1,0$ and +1 , respectively. A total of 9 experiments and the addition of a central point interspersed among the experimental setting runs were considered. The latter provided a measure of the process stability or intrinsic variability, and allowed evaluating the model curvature, and the quadratic relationship between the selected response $(\mathrm{Y})$ and each of the factors $\left(\mathrm{x}_{1}, \mathrm{x}_{2}\right)$, Eq. (1)

$Y=\beta_{0}+\beta_{1} x_{1}+\beta_{2} x_{2}+\beta_{12} x_{1} x_{2}+\beta_{11} x_{11}^{2}+\beta_{22} x_{22}^{2}+\varepsilon$

where $\mathrm{Y}$ is the measured response for the dependent variables associated to each factor level combination, $\beta_{0}$ is the arithmetic mean of the experiments, $\beta_{1}$ and $\beta_{2}$ are the regression coefficients of the respective factors, $\beta_{12}$ is the interaction coefficient between the two factors, $\beta_{11}$ and $\beta_{22}$ are the quadratic coefficients obtained from the observed experimental values of $\mathrm{Y}, \mathrm{x}_{1}$ and $\mathrm{x}_{2}$ are the terms representing the two factors, $\mathrm{x}_{11}$ and $\mathrm{x}_{22}$ represent the quadratic terms, $\mathrm{x}_{1} \mathrm{x}_{2}$ corresponds to the interaction term, while $\varepsilon$ is the regression error.

$2^{\mathrm{k}}$ full factorial design

The second design was also used to explain the effects of the amount of aqueous surfactant and the production method on PS, PI and ZP. For usNLCs containing a solid:liquid lipid ratio of 25:75 (see Results and Discussion, section 3.3.2), the concentration of aqueous surfactant Tween ${ }^{\circledR} 80$ (factor 1 ) was imposed as 1 and $5 \%(\mathrm{w} / \mathrm{w})$, corresponding to the coded levels -1 and +1 , respectively. A categorical factor (factor 2 ) based on two different production methods, US (-1) and HPH $(+1)$ was also defined. The regression model Eq. (2)

$Y=\beta_{0}+\beta_{1} x_{1}+\beta_{2} x_{2}+\beta_{12} x_{1} x_{2}+\varepsilon$

was defined considering 4 experiments.

\subsection{Physicochemical characterization of nanocarriers}

\subsubsection{Particle size, polydispersity index and zeta potential}

The mean PS and PI were measured by dynamic light scattering (DLS) with a detection angle of $173^{\circ}$ at $25^{\circ} \mathrm{C}$. The PS is calculated from the translational diffusion coefficient by employing the Stokes-Einstein equation. The cumulants method was used for data analysis. ZP was determined by electrophoretic light scattering (ELS) set at a temperature of $25{ }^{\circ} \mathrm{C}$. The Helmholtz-Smoluchowsky equation was used for the zeta potential calculation. All the parameters were measured using a Zetasizer Nano ZS (Malvern Instruments, Malvern, UK). The samples were subjected to a 100 -fold dilution with ultra-purified water and analyzed in triplicate. Results are presented as mean \pm standard deviation. The physicochemical stability of NLCs with 25:75, 50:50 and 75:25 solid:liquid lipid ratios was evaluated measuring PS, PI and ZP at $1,7,30,60,90$ and 180 days after production.

\subsubsection{Entrapment efficiency and drug loading}

EE and DL of both ATO and CUR in the NLCs were calculated according to the following equations Eqs. (3) and (4)

$\% E E=\left(W_{\text {total drug }}-W_{\text {free drug }}\right) / W_{\text {total drug }} \times 100$

$\% D L=\left(W_{\text {total drug }}-W_{\text {free drug }}\right) / W_{\text {lipid }} \times 100$

where $\mathrm{W}_{\text {total drug }}$ is the amount of drugs determined in the whole nanosystem, $W_{\text {free drug }}$ is the amount of free drugs determined in the aqueous phase after ultrafiltration-centrifugation of the NLCs, and $\mathrm{W}_{\text {lipid }}$ is the weight of the lipid phase of the NLCs.

The quantification of ATO and CUR was performed using a HPLC method previously validated according to FDA and ICH recommendations (Basso et al., 2018). A Shimadzu LC-2010HT apparatus equipped with a quaternary pump (LC-20AD), an auto-sampler unit (SIL-20AHT), a CTO-10AS oven and a SPD-M2OA detector was used. The chromatographic analyses were performed on a Kinetex ${ }^{\circledR}$ EVO C18 column (Torrance, USA), with $5 \mu \mathrm{m}$ particle size, $4.6 \mu \mathrm{m}$ internal diameter and $150 \mathrm{~mm}$ length, at $35^{\circ} \mathrm{C}$. The mobile phase was composed of a mixture of $2 \%(\mathrm{v} / \mathrm{v})$ glacial acetic acid:acetonitrile $(50: 50, \mathrm{v} / \mathrm{v})$ and was eluted at an isocratic flow rate of $1.0 \mathrm{~mL} / \mathrm{min}$. A run time of 6 min was established, with CUR eluting at $4.5 \mathrm{~min}$ and ATO at $5.1 \mathrm{~min}$. The UV-Vis detection was carried out at $425 \mathrm{~nm}$ and $247 \mathrm{~nm}$ for CUR and ATO, respectively, and an injection volume of $10 \mu \mathrm{L}$ was used for all samples. The results were processed using the Shimadzu LC-solution version 1.12 software.

\subsubsection{In vitro release assay}

In vitro release studies of ATO and CUR from the NLCs containing 25:75, 50:50 and 75:25 solid:liquid lipid ratios and the reference solution $(3.75 \mathrm{mg} / \mathrm{mL}$ of ATO and $1.875 \mathrm{mg} / \mathrm{mL}$ of CUR) in PEG 400 were performed using a dialysis cellulose membrane $(\mathrm{MWCO} \approx 14.000$, avg, flat width $33 \mathrm{~mm}$, D9652, Sigma-Aldrich), as artificial membrane. In order to ensure sink conditions, the release medium is composed of $30 \%$ $(\mathrm{V} / \mathrm{V})$ of PEG 400 and $70 \%(\mathrm{~V} / \mathrm{V})$ of phosphate buffered saline (PBS, $\mathrm{pH}=7.4$ ). A volume of $3 \mathrm{~mL}$ containing a known amount of ATO and CUR in NLCs was placed inside the dialysis membrane, and both ends were tied to prevent leakage. The membrane was dipped into $100 \mathrm{~mL}$ of the release medium, and the medium was stirred continuously at $50 \mathrm{rpm}$, maintaining the temperature at $37{ }^{\circ} \mathrm{C} \pm 0.5{ }^{\circ} \mathrm{C}$. A volume of $150 \mu \mathrm{L}$ of each sample was withdrawn at predetermined intervals $(0.5$, $1,3,6,9,12,24,30,36$ and $48 \mathrm{~h}$ ) and immediately replenished with fresh medium. The samples were diluted in mobile phase and drug release was analyzed using the HPLC method aforementioned. In order to investigate the differences between the ATO and CUR release profiles obtained from the corresponding formulations, the similarity factor $f_{2}$ was determined, taking into account the criteria mentioned in the EMA guideline (EMA, 2010). It was calculated according to Eq. (5)

$f_{2}=50 \times \log \left[\frac{100}{\sqrt{1+\frac{\sum_{t=1}^{t=n}[\bar{R}(t)-\bar{T}(t)]^{2}}{n}}}\right]$

where $f_{2}$ is the similarity factor, $n$ is the number of time points, $\bar{R}(t)$ is the mean percent reference drug dissolved at time $t$ after initiating the study, $T(t)$ is the mean percent test drug dissolved at time $t$. Also, the release patterns of ATO and CUR were fitted to different mathematical models (zero order, first order, Higuchi, Weibull and KorsemeyerPeppas equations), aiming at identifying the optimal mathematical function describing the kinetics of the drug release process. 

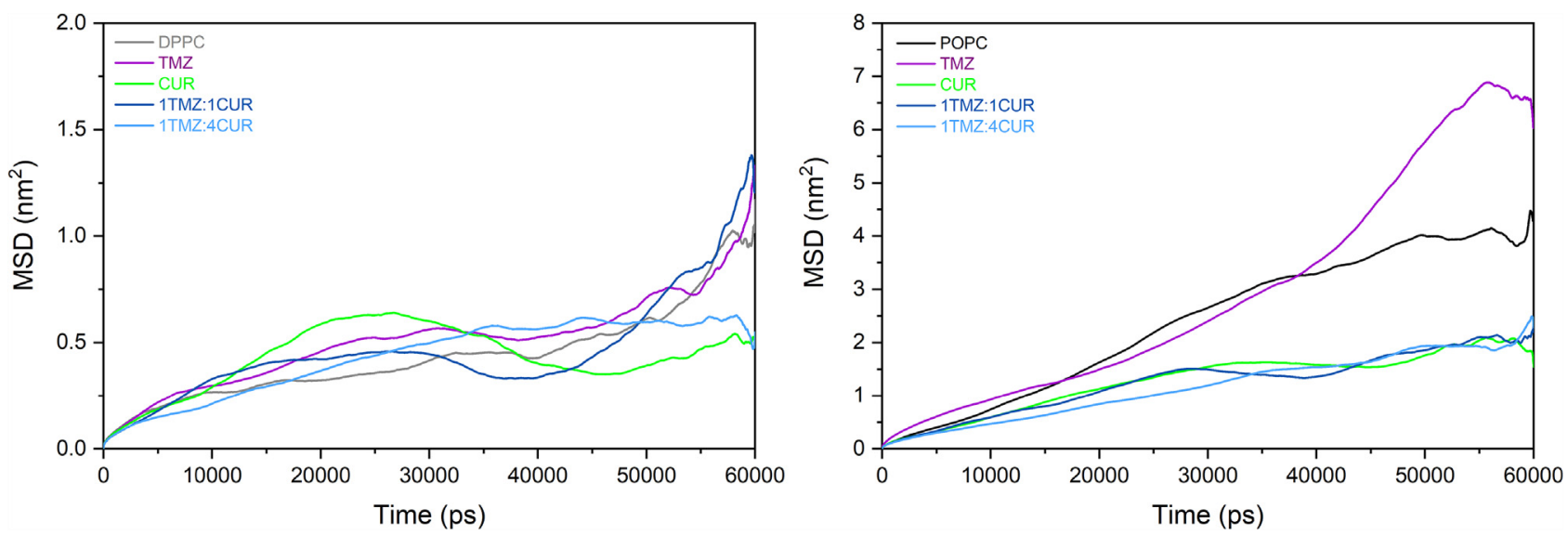

Fig. 2. Mean square displacement (MSD) profiles of DPPC and POPC, along the simulation runs for DPPC/TMZ, DPPC/CUR, POPC/TMZ and POPC/CUR systems.

\subsection{RBC-cloaked usNLCs}

The RBC vesicles were prepared following a previously reported procedure (Hu et al., 2011). Briefly, whole blood from a healthy human donor was collected and $1 \mathrm{~mL}$ centrifuged at $800 \times \mathrm{g}$ for $5 \mathrm{~min}$ at $4{ }^{\circ} \mathrm{C}$ before the removal of the serum and buffy coat. The resulting RBCs were washed once in ice cold PBS and submitted to hypotonic treatment. RBC ghosts were then also washed once with ice cold PBS until the removal of hemoglobin, sonicated in a glass vial for $5 \mathrm{~min}$ and extruded 7 times through a $100 \mathrm{~nm}$ polycarbonate porous membrane (Sigma, USA) to obtain the RBC vesicles. To fuse the vesicles with the nanoparticles, $0.5 \mathrm{~mL}$ of usNLCs were mixed with the previously obtained vesicles and extruded through a $100 \mathrm{~nm}$ polycarbonate porous membrane.

In order to investigate and optimize the coating procedure, a Taguchi $\mathrm{L}_{9}, 3^{3}$ orthogonal array design was performed using $\mathrm{JMP}^{\circledR}$ Pro software, version 14.0.0 (SAS Institute Inc., USA). According to the design matrix, a total of 9 experiments were conducted. Three independent continuous factors were considered at three levels $(-1,0,1)$, namely the number of co-extrusions (3, 9 and 15), the volume of RBC vesicles $(50,100$ and $150 \mu \mathrm{L})$ and the concentration of stearylamine $(0$, 1 and $2 \mathrm{mM}$ ), a cationic lipid added during production to variate the surface charge of the lipid core. The three factors were studied accordingly and their impact on PS, PI and ZP was assessed. The S/N ratios were calculated with

$S / N$ ratio $=-10 \log \left[\left(\sum y^{2}\right) / n\right]$

Eq. (6) and analyzed under the "lower is the better" condition, aiming to understand factors effect. A lower $\mathrm{S} / \mathrm{N}$ ratio infers a higher impact of the respective parameter on the response. In Eq. (6), $n$ is the number of observations and $y$ is the observed data for the respective response. The physicochemical properties were measured as previously described in Section 2.6.1.

\subsection{Statistical data analysis}

One-way analysis of variance (ANOVA) and Student's $t$-test ( $\alpha=0.05$ ) were performed for assessing the statistical significance and the magnitude of the effects of the formulation variables, and the respective coefficients of the regression model ( $\mathrm{p}<0.05$ ). A two-way ANOVA ( $\alpha=0.05$ ) with Tukey's multiple comparison test was also employed using PRISM 7 (GraphPad software, USA), in order to assess the statistical significance of the differences among NLCs $(\mathrm{p}<0.05)$.

\section{Results and Discussion}

\subsection{Repurposing drugs}

\subsubsection{Selecting the $B B B$ mimicking membrane}

In order to select a simple phospholipid membrane model for BBB and to explore the interaction of TMZ and CUR with the BBB, single TMZ and CUR molecules, as well as different proportions of both molecules, were introduced into two different phospholipid bilayers, DPPC and POPC.

The impact of TMZ and CUR incorporation on the structural characteristics of DPPC and POPC bilayers were firstly assessed using the density profiles depicted in Fig. S1 (Supplementary Material). These density profiles represent the relative positioning of the key atom groups pertaining to the phospholipids and the drugs relatively to the bilayer center, defined as the region between the membrane leaflets. For the analysis of DPPC and POPC, the P and $\mathrm{N}$ atoms of the lipid head groups, and the terminal methyl groups of the hydrocarbon tails were selected. TMZ and CUR molecules were represented by the respective center of mass. From panels A and D, it can be seen that TMZ is preferentially positioned close to the interface, due to the respective hydrophilic character. On the other hand, a slight internalization of CUR, displaying a hydrophobic character, towards the interior of the bilayers is observed. (Fig. S1, panels B and E, Supplementary Material). In the presence of CUR (Fig. S1, panels C and F, Supplementary Material), a 1:1 complex with TMZ is formed, promoting the internalization of TMZ into both DPPC and POPC, with a more pronounced effect for the latter. Despite of the hydrophilic nature of TMZ, it is desirable that this drug permeates the membrane in order to exert its therapeutic effects at the brain level. Permeation ability of TMZ can be enhanced by the association with CUR, especially when considering the POPC bilayer.

Further analyses based on the ordering of hydrocarbon tails of the bilayers were performed by estimating the deuterium order parameter $\left(\mathrm{S}_{\mathrm{CD}}\right)$ along the DPPC and POPC chains in the presence of TMZ and CUR. The results are displayed in Fig. S2 (Supplementary Material). For DPPC systems, all compounds induce a disordering effect along the alkyl chains of the bilayer, while a greater variability is observed for POPC. The association of TMZ and CUR (1:1) induced a lower perturbation of POPC.

Fig. 2 shows the mean square displacement (MSD) profiles suggesting slight differences in terms of the lateral diffusion of the DPPC bilayer after the incorporation of single, 1TMZ:1CUR and 1TMZ:4CUR. However, the introduction of TMZ into POPC promotes the highest lateral diffusion of the lipid molecules. This suggests that a higher membrane perturbation is obtained in POPC, which was then selected as membrane model to proceed with the analyses and study the behavior of BBB and glioblastoma cell membranes. 


\subsubsection{Finding the best statin}

The potential repositioning of different statin generations, including atorvastatin (A), atorvastatin calcium (ATO), pitavastatin (P), pitavastatin calcium (PIT) and simvastatin (SV), for glioblastoma treatment, is explored in what follows, considering the POPC as membrane model of the BBB. Fig. S3 (Supplementary Material) presents the density profiles of the statins in POPC bilayers. Different stoichiometry was considered for systems containing the salt forms of $A$ and $P$. In these cases, for one calcium ion $\left(\mathrm{Ca}^{2+}\right)$, two molecules of both $\mathrm{A}$ and $\mathrm{P}$ were considered, so that the systems have neutral charge. Statins are in general embedded in the hydrophobic region of the bilayer. However, ATO and PIT are positioned close to the interface. The calcium salts are more hydrophilic than the neutral molecules, which restrains the mobility of the former to the hydrophilic regions of the membrane, i.e. close to the phospholipid polar heads. This is also suggested by the solvation profiles of each statin, obtained from the radial distribution function (RDF) of water molecules around the main hydrophilic groups of statins, as presented in Fig. S4 (Supplementary Material). These profiles suggest an increased solvation effect of ATO and PIT (panels B and D, respectively), while the other three statins, A, P and SV (panels A, C and E, respectively) are not solvated, corroborating the respective density profiles, and thus indicating a deeper internalization of $\mathrm{A}, \mathrm{P}$ and $\mathrm{SV}$ in the POPC membrane.

Fig. 3 and Table 1 present, respectively, the MSD profiles and the diffusion coefficients for each compound, indicating significant structural modifications of POPC. The incorporation of ATO is responsible for a significant increase in the lateral diffusion of the bilayer, when compared to neat POPC, and, consequently, ATO exhibited the higher diffusion coefficient. A decreased lateral diffusion of the POPC molecules is found in the presence of PIT and the lowest diffusion coefficient was obtained for P. In general, ATO appears to gather the most attractive properties, i.e. solubility, $\log \mathrm{P}$ and permeability to cross the membrane, and to proceed with the comparison of membrane crossabilities between ATO and the standard of care in glioblastoma, TMZ.

Two systems containing different proportions of TMZ and ATO were considered to evaluate the behavior of both molecules into POPC and infer on the respective ability to cross biological membranes. From the density profiles and RDF analysis (Fig. S5 and 4), it can be seen that TMZ and ATO tend to be positioned closer to the interface. However, an increased number of water molecules in the proximity of TMZ is observed, considering the amine and carbonyl groups, followed by the diazo groups. For ATO, the carboxyl groups are clearly solvated, while the hydroxyl groups are not solvated. This is in agreement with the previous density profiles which suggested that ATO displays an amphiphilic character, as it tends to be placed in both hydrophobic and hydrophilic regions of the membrane. Similar observations are found from MSD (Fig. 5), which indicate an increased lateral diffusion of the POPC membrane for both ATO and TMZ.

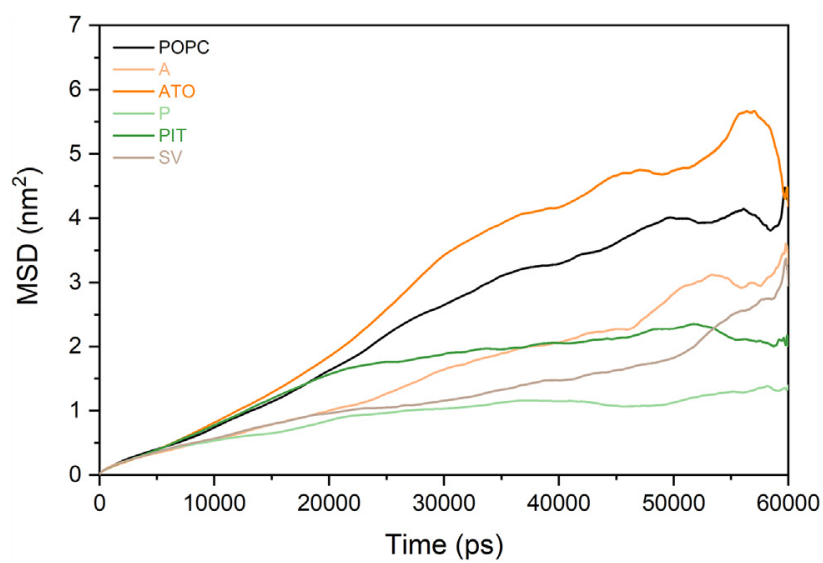

Fig. 3. Mean square displacement (MSD) of POPC molecules, calculated from MD for the POPC/A, POPC/ATO, POPC/P, POPC/PIT and POPC/SV systems.
Table 1

Diffusion coefficients of POPC, temozolomide, curcumin and the different statins obtained from mean square displacement (MSD).

\begin{tabular}{ll}
\hline System & Diffusion coefficient $\left(\mathrm{nm}^{2} / \mathrm{ps}\right)$ \\
\hline Temozolomide & $0.0289 \pm 0.0282$ \\
Curcumin & $0.0069 \pm 0.0097$ \\
Atorvastatin & $0.0082 \pm 0.0013$ \\
Atorvastatin calcium & $0.0185 \pm 0.0099$ \\
Pitavastatin & $0.0054 \pm 0.0024$ \\
Pitavastatin calcium & $0.0112 \pm 0.0059$ \\
Simvastatin & $0.0061 \pm 0.0046$ \\
POPC & $0.0071 \pm 0.0008$ \\
\hline
\end{tabular}

It is apparent that ATO exhibits a diffusion behavior similar to TMZ $\left(0.0185 \pm 0.0099 \mathrm{~nm}^{2} / \mathrm{ps}\right.$ versus $0.0289 \pm 0.0282 \mathrm{~nm}^{2} / \mathrm{ps}$, respectively) with the advantage of displaying a higher log $\mathrm{P}$ value $(5.32$ versus -1.15 for TMZ (46), and therefore, an increased ability to penetrate the hydrophobic region of lipid bilayers. Based on membrane perturbation effects, ATO was selected for mimicking the TMZ diffusion behavior, and to be used as a repurposed drug in glioblastoma.

\subsubsection{Assessing the effect of curcumin}

The potential synergism among ATO and CUR was also evaluated. As previously observed, TMZ interacts more strongly with the polar heads of POPC (Fig. S6, panel A, Supplementary Material), while ATO and CUR are slightly internalized into the bilayer (Fig. S6, panels B and C, respectively, Supplementary Material). Additionally, to compare the cross-membrane ability of TMZ/CUR and ATO/CUR conjugates, two systems were constructed with the compounds in the same proportions (2:2). In the 2TMZ:2CUR system (Fig. S6, panel D) TMZ and CUR tend to be positioned close to the interface, interacting more strongly with the lipid polar heads. On the other hand, in the system containing 2ATO:2CUR (Fig. S6, panel E, Supplementary Material), the molecules are more embedded in the hydrophobic region of the bilayer, suggesting an enhanced permeation effect. This is also evident in Fig. 6, which shows four representative configurations of single TMZ and ATO, and combined TMZ/CUR and ATO/CUR, and in the solvation profiles of Fig. 7. From Fig. 8, it is possible to infer that only TMZ and ATO are able to increase the lateral diffusion of POPC, when compared with neat POPC. The lateral diffusion of the POPC molecules is reduced in the presence of 2ATOC:2CUR, due to the enhanced hydrophobicity and membrane permeability induced by CUR, which may also improve the encapsulation properties into lipid nanoparticles.

Taking into account the previous permeation pattern into POPC bilayer, ATO and CUR, were then selected to proceed with the experimental studies regarding the development and optimization of NLCs. Further, the best proportion between the two therapeutic agents was chosen based on their solubility.

\subsection{Evaluating risk}

The potential cause-and-effect relationships among the key material and process variables that exhibit a great impact on the NLCs quality were highlighted in the Ishikawa diagram of Fig. 9. Once the CMAs and CPPs that may influence the CQAs variability were identified, a RAM was constructed according to their potential criticality in terms of risk (Table 2). RAM suggests that the solid:liquid lipid ratio and surfactant concentration are the key CMAs influencing the quality of the nanocontructs, due to the high risk associated with them on most CQAs. The drug solubility, and lipid and drug concentration display a high-risk level for EE and DL, and, additionally, the drug solubility is a high-risk CMA for drug release. Also, the homogenization method type (CPP) was associated with high-risk levels assigned to PS and PI and with mediumrisk levels ascribed to ZP. Thus, based on the results of the risk assessment, the solid:liquid lipid ratio, surfactant concentration and type 

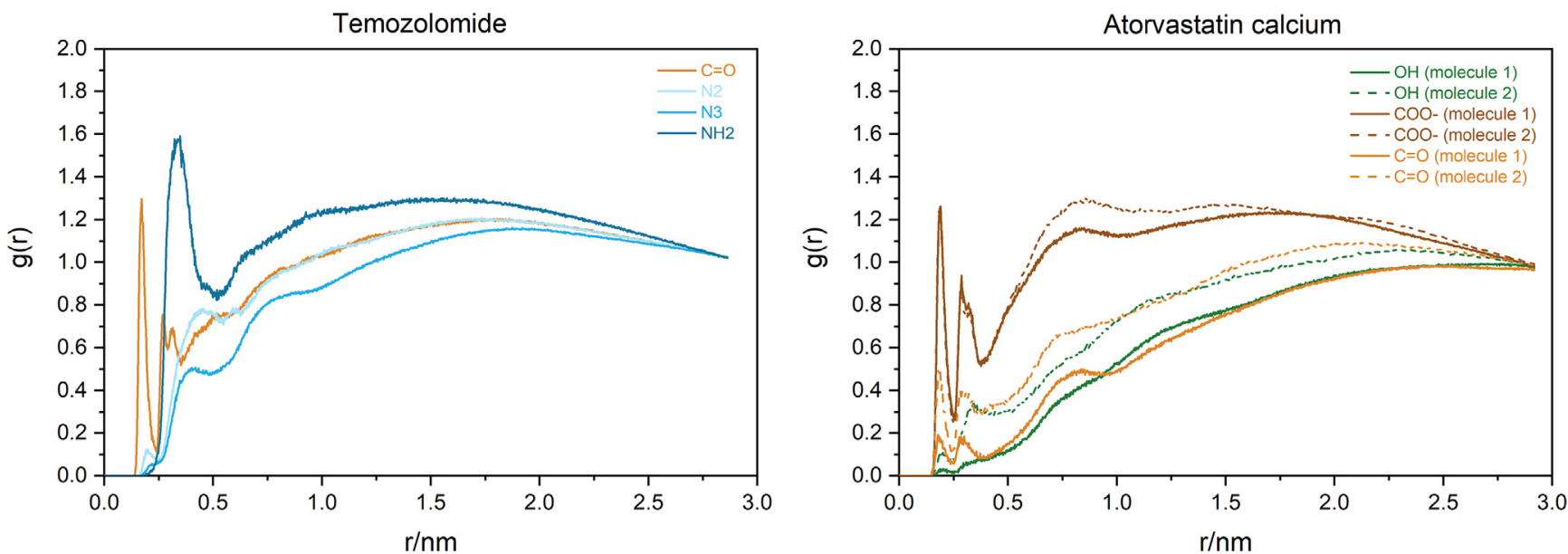

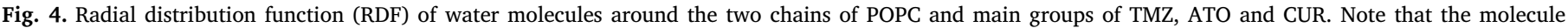
identification refers to the single molecules that composed calcium salts.

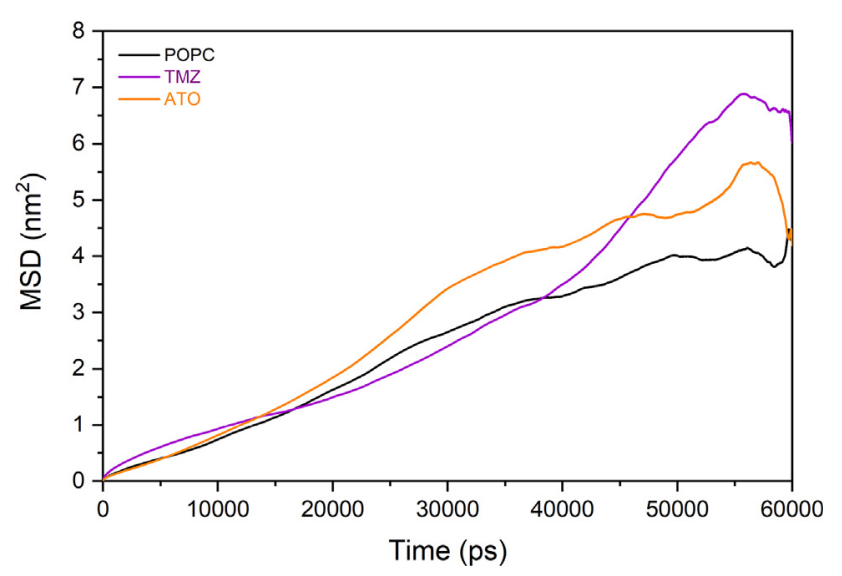

Fig. 5. Mean square displacement (MSD) of POPC molecules, calculated from the molecular dynamics simulations for the POPC/TMZ and POPC/ATO systems.

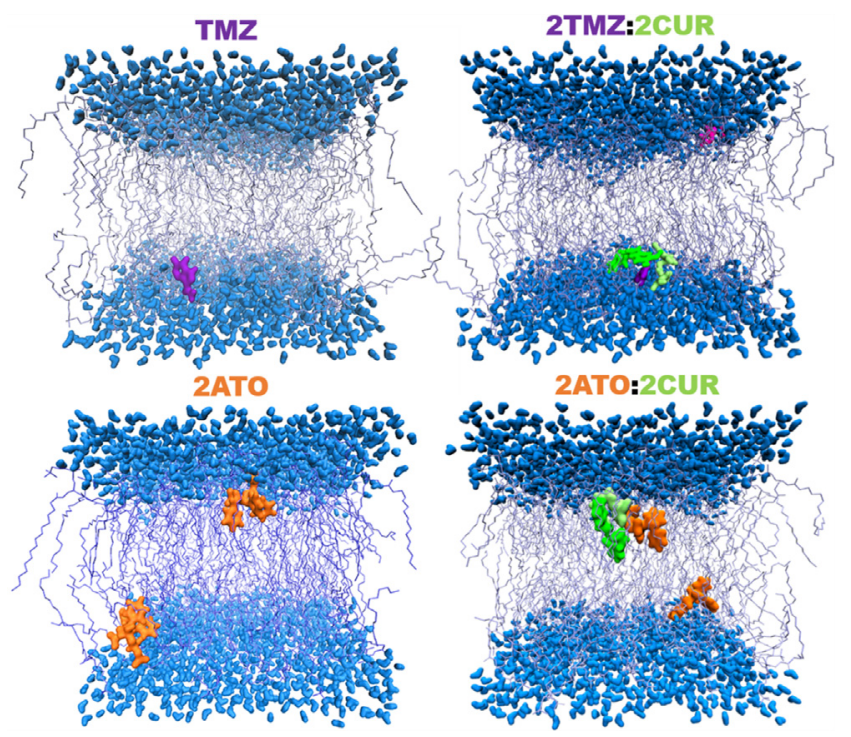

Fig. 6. Representative snapshots illustrating the relative preferential positioning of TMZ, ATO and CUR embedded in the POPC bilayer, considering different proportions of TMZ, ATO and CUR: single TMZ, 2TMZ:2CUR, 2ATO and 2ATO:2CUR. Water molecules, TMZ, ATO, CUR and POPC are represented in blue, purple, orange, green and steelblue, respectively. of the production method were selected as the most critical parameters influencing the performance of the NLCs, and were further investigated in the following DoE studies. Moreover, the qualitative effect of the type and concentration of lipid and surfactant were evaluated through multivariate analysis methods for an initial screening, in order to reduce the variables to be studied in the experimental step.

\subsection{Optimizing usNLC}

\subsubsection{Selecting raw materials}

As a preliminary screening in the optimization step, a set of twentyfive ATO and CUR co-encapsulated NLCs was firstly produced by high shear homogenization, followed by ultrasonication, where several changes in the composition and concentration of the liquid lipids and oily surfactants were evaluated. Tween ${ }^{\circledR} 80$ (T80) was used as the aqueous surfactant due to its intrinsic properties that make it a potential strategic excipient for brain targeting, in particular: (i) the solubilization of the endothelial cell membrane lipids and membrane fluidization, (ii) the adsorption of certain plasma apolipoproteins (e.g. ApoE and ApoB) onto the NLCs surface, promoting their LDL receptor-mediated endocytosis into the brain (due to overexpression on BBB endothelial cells), and (iii) the inhibition of the efflux function of P-gp (Kaur et al., 2008; Neves et al., 2015; Gelperina et al., 2010; Göppert and Müller, 2005). The same rational was considered for the selection of cetyl palmitate as solid lipid, which has also been shown to adsorb many plasma apolipoproteins (e.g. ApoA-I, ApoC-III and ApoJ), prompting the NLCs transport into the brain. Additionally, it allows to obtain suitable NLC sizes $(<200 \mathrm{~nm}$ ) for brain targeting by intravenous administration (Miranda et al., 2017; Gupta et al., 2017; Blasi et al., 2011). The characterization of these NLCs was performed and the obtained results for physicochemical properties (PS, PI and ZP) are depicted in Table 3. NLCs with very different PS, PI and ZP values were obtained, being comprised between 53.4 and $1684 \mathrm{~nm}, 0.150$ and 0.680 , and -35.97 and $-7.2 \mathrm{mV}$, respectively. As previously described in the risk assessment, the pre-emulsion composition and process parameters affect the properties of the NLCs, significantly impacting the quality of the final product (Lason et al., 2013). Hence, the choice of the variables is critical and must be carefully carried out. PS shows a key role in determining biodistribution of the nanoparticles after being injected in vivo and in simplifying their access to BBB and tumor cells. It is well defined that particles less than approximately $100 \mathrm{~nm}$ in diameter extravagate favorably from the tumor capillary vasculature, due to a better vascular permeability compared with normal tissue and are retained in the tumor tissue rather than returning freely to the circulation (Perrault et al., 2009). ZP also denotes an important parameter that 

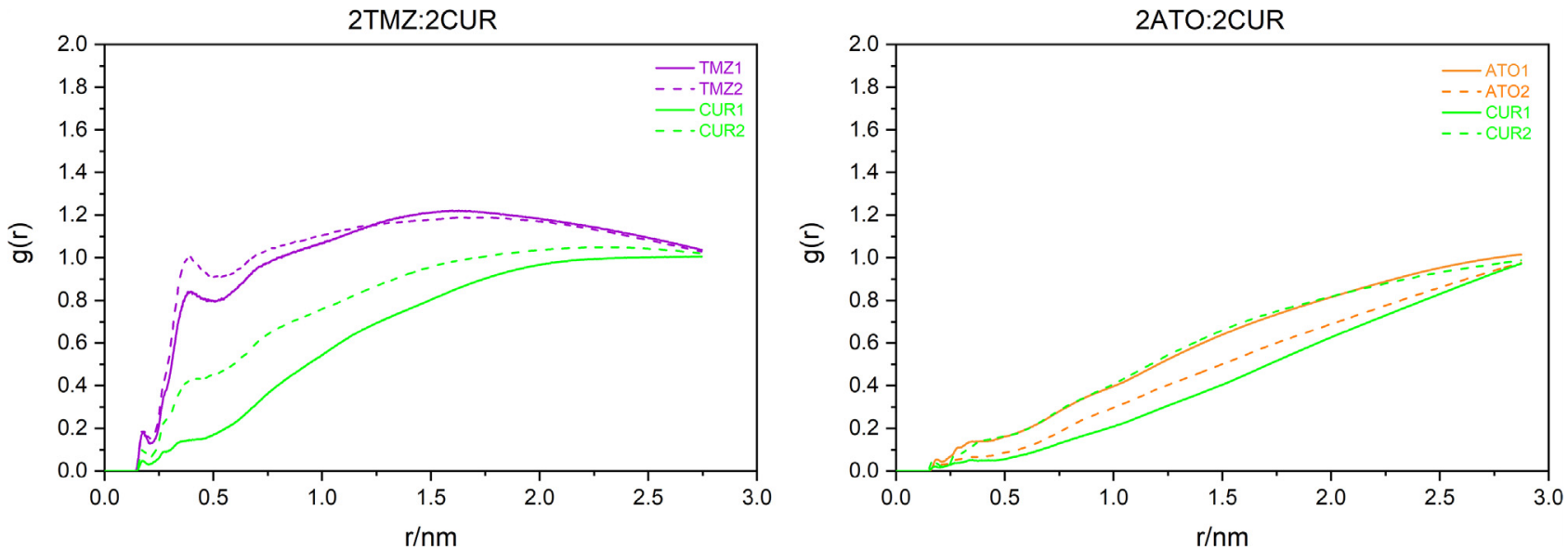

Fig. 7. Radial distribution function (RDF) of water molecules around the two chains of POPC and the main groups of TMZ, ATO and CUR in $2 \mathrm{TMZ}: 2 \mathrm{CUR}$ and 2ATO:2CUR systems. TMZ, ATO and CUR were represented by the respective center of mass.

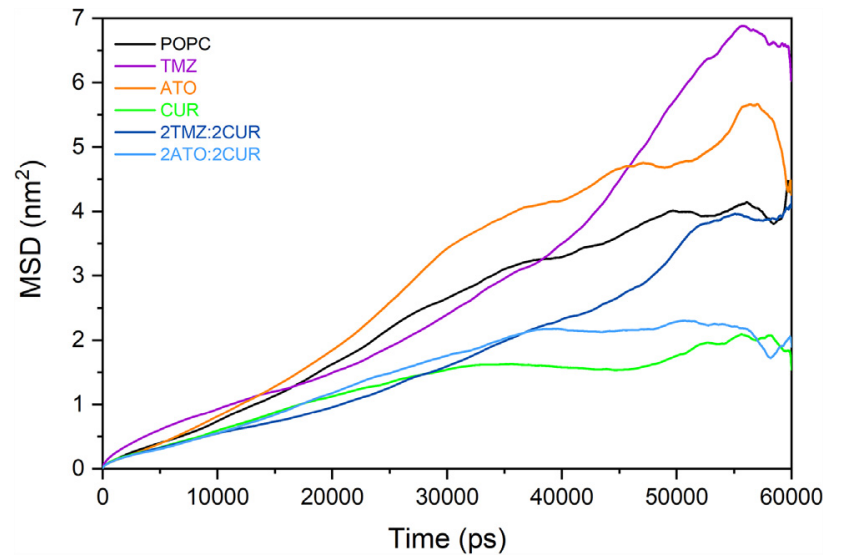

Fig. 8. Mean square displacement (MSD) of POPC in the presence of TMZ, ATO and CUR, in separate and in combination.

influences long-term stability of colloidal systems. NLCs possessing a ZP value higher than $|30| \mathrm{mV}$ are strongly charged and, consequently, physically stable due to the particle-particle repulsion (Pourgholi et al., 2016). Consequently, in this study it is intended to develop a prototype formulation based on small, homogenous and stable NLCs.

The results presented in Table 3 were analyzed employing PCA and PLS, in order to investigate the effect of the NLCs composition on PS, PI and ZP. The focus of this preliminary screening was the influence of the type and amount of liquid lipids, Capryol ${ }^{\mathrm{TM}}$ PGMC (C), Transcutol ${ }^{\circledR} \mathrm{HP}$ (T) and Labrasol ${ }^{\circledR}$ (L), and oily surfactants, Lipoid S 75 (LS75) and Lipoid S PC 3 (LSPC3). Fig. 10 depicts the biplot representation of the twenty-five developed formulations (Table 3 ) on the first two principal components, PC1 and PC2, corresponding to ca. $57.8 \%$ of data variability. NLCs and their characteristics are represented by points (scores) and vectors (loadings), respectively.

Along the first two PCs, the liquid lipids $\mathrm{C}$ and $\mathrm{T}$ are positively correlated, but these are, in turn, negatively correlated with the lipid $\mathrm{L}$. The oily surfactants LS75 and LSPC3 are also negatively correlated. Pertaining to the three measured parameters of NLCs, they are positively correlated on PC1, but PS and PI are negatively correlated with ZP along PC2. Therefore, the NLC excipients have a different effect on these parameters. The optimal composition that allows the production of small and homogeneous NLCs is achieved with the presence of liquid lipids $\mathrm{C}$ and $\mathrm{T}$, which are located in the opposite region relatively to PS and PI, considering PC1 and PC2. Inversely, the lipid L is directly and strongly correlated with PS and PI, leading to NLCs with higher values of these parameters. Note that these lipids have similar correlation with ZP along PC1, but an inverse expression is identified along PC2. Regarding the studied oily surfactants, they have a greater effect on ZP than on PS and PI, along PC1 and PC2. The surfactant LS75 is negatively correlated with ZP, so the NLCs that contain LS75 are characterized by low values of this parameter, i.e. present more negative values of ZP. On the other hand, the surfactant LSPC3 is directly correlated with ZP, leading to high values of this parameter, i.e. negative values closer to zero. Along PC1, the LS75 and LSPC3 surfactants are direct and inversely correlated with PS and PI. Thus, the influence of the type of oily surfactant on the PS and PI parameters is not clearly evidenced. This representation and interpretation is very useful to determine and control the composition of NLCs and characterize them, by an easy and rapid evaluation of NLC scores and variable loadings. For instance, without a prior knowledge, it is possible to deduce that the NLCs

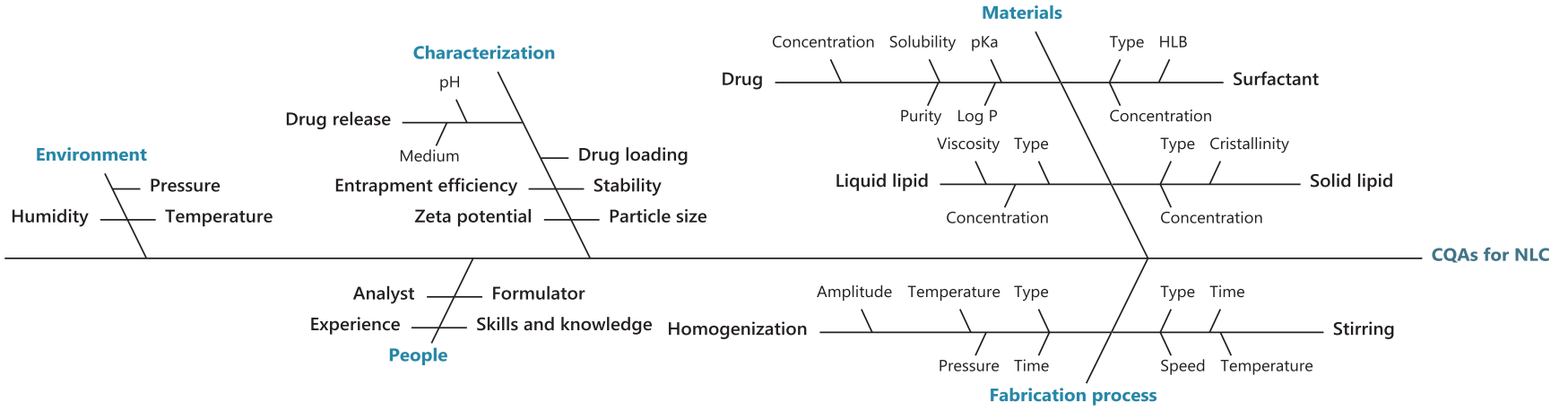

Fig. 9. Ishikawa diagram representing the cause-and-effect relationship among the critical material and process variables for the critical quality attributes (CQAs) of NLCs. 
Table 2

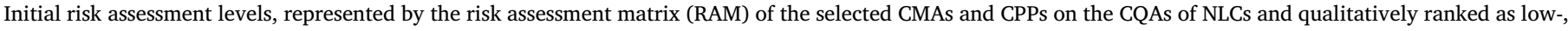
medium- or high-risk.

\begin{tabular}{|c|c|c|c|c|c|c|c|c|}
\hline \multirow[t]{2}{*}{ CQAs } & \multicolumn{7}{|l|}{ CMAs } & \multirow{2}{*}{$\begin{array}{l}\text { CPPs } \\
\begin{array}{l}\text { Homogenization } \\
\text { method type }\end{array}\end{array}$} \\
\hline & $\begin{array}{l}\text { Type of } \\
\text { lipid }\end{array}$ & $\begin{array}{l}\text { Lipid } \\
\text { concentration }\end{array}$ & $\begin{array}{l}\text { Solid:liquid lipid } \\
\text { ratio }\end{array}$ & $\begin{array}{l}\text { Type of } \\
\text { surfactant }\end{array}$ & $\begin{array}{l}\text { Surfactant } \\
\text { concentration }\end{array}$ & $\begin{array}{l}\text { Drug } \\
\text { concentration }\end{array}$ & $\begin{array}{l}\text { Drug } \\
\text { solubility }\end{array}$ & \\
\hline PS & Medium & Medium & High & Medium & High & Medium & Low & High \\
\hline PI & Low & Low & Medium & Medium & High & Medium & Low & High \\
\hline $\mathrm{ZP}$ & Medium & Medium & Medium & High & High & Medium & Low & Medium \\
\hline $\mathrm{EE}$ & Medium & High & High & Medium & Medium & High & High & Low \\
\hline DL & Medium & High & High & Low & Medium & High & High & Low \\
\hline Drug release & Medium & Medium & High & Medium & High & Medium & High & Low \\
\hline
\end{tabular}

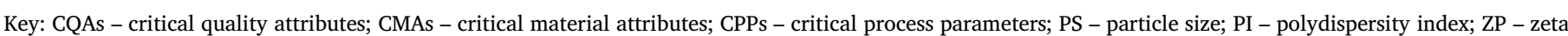
potential; EE - entrapment efficiency; DL - drug loading.

represented by the number 3 are only composed by the lipid L, while those represented by the numbers 18 and 23 are constituted by the lipid L and surfactant LSPC3, but NLCs 23 present a higher concentration of this surfactant than the NLC 18. Furthermore, these NLCs are characterized by high PS and PI values and intermediate ZP values.

In order to complement PCA results, the PLS method was also employed. The PLS models of PS, PI and ZP, considering two latent variables, explain $46.82 \%, 19.12 \%$ and $72.58 \%$ of the variance of these responses, respectively, and the obtained regression coefficients are depicted in Fig. 11. Analyzing the value and the signal of these coefficients, it is possible to infer that the lipids $\mathrm{C}$ and $\mathrm{T}$ and the surfactant LS75 show an inverse effect on PS, i.e. are inversely correlated, expressed by negative coefficients. Thus, their presence and a high concentration of these components leads to NLCs with small PS. In turn, the remaining variables, i.e. the lipid L and the surfactant LSPC3, have a direct effect on PS, leading to NLCs with high PS values. Regarding the $\mathrm{PI}$, the lipid C is inversely correlated with this parameter, presenting a negative coefficient, while the other two selected lipids express positive coefficients. Furthermore, the considered oily surfactants are directly correlated with PI. Thus, the presence and a high concentration of the lipid L and these two surfactants yields more heterogeneous NLCs. The
ZP is directly affected by the presence and high concentrations of the lipid T, which leads to slightly higher ZP values, as verified by the positive but low coefficient. On the other hand, all the remaining variables present negative coefficients, with the surfactant LS75 being represented by a more negative coefficient and, consequently, having a higher inverse effect on ZP, i.e. is in agreement with low ZP values. Similar observations were obtained with PCA.

PCA and PLS results suggest that the components that have a significant impact on the quality of NLCs are the lipid C and the surfactant LS75. These are the components that allow obtaining smaller PS, relatively low PI and more negative ZP values. However, considering the drug solubility as an important CMA that influences other properties, such as EE, DL and drug release, it is possible to conclude that the lipid $\mathrm{C}$ is not adequate due to the low ATO and CUR solubility in this lipid (ATO: $18 \pm 3 \mathrm{mg} / \mathrm{mL}$ (Elmowafy et al., 2017); CUR: $4.5 \pm 0.7 \mathrm{mg}$ / $\mathrm{mL}$ ). Thus, the lipids $\mathrm{T}$ and $\mathrm{L}$ were selected to proceed with the design of optimal NLCs, as the solubility values in T (ATO: $242 \pm 3 \mathrm{mg} / \mathrm{mL}$ (Kassem et al., 2017); CUR: $77 \pm 15 \mathrm{mg} / \mathrm{mL}$ ) and L (ATO: $89 \pm 9 \mathrm{mg} / \mathrm{mL}$; CUR: $44.2 \pm 0.6 \mathrm{mg} / \mathrm{mL}$ ) are quite satisfactory.

Table 3

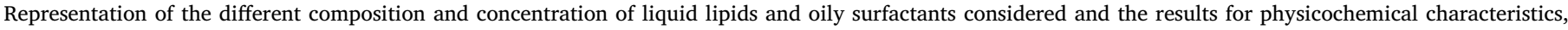
namely PS, PI and ZP, of ATO and CUR co-encapsulated NLCs produced by ultrasonication.

\begin{tabular}{|c|c|c|c|c|c|c|c|c|c|}
\hline Formulation & Code & $\mathrm{C}$ & $\mathrm{T}$ & $\mathrm{L}$ & LS75 & LSPC3 & PS (nm) & PI & $\mathrm{ZP}(\mathrm{mV})$ \\
\hline $\operatorname{PrC}$ & 1 & 100 & 0 & 0 & 0 & 0 & $385 \pm 7$ & 0.180 & $-13 \pm 0$ \\
\hline PrT & 2 & 0 & 100 & 0 & 0 & 0 & $139 \pm 1$ & 0.300 & $-13 \pm 1$ \\
\hline PrL & 3 & 0 & 0 & 100 & 0 & 0 & $536 \pm 17$ & 0.480 & $-9.4 \pm 0.3$ \\
\hline PrTC & 4 & 50 & 50 & 0 & 0 & 0 & $183 \pm 2$ & 0.300 & $-12 \pm 1$ \\
\hline PrTL & 5 & 0 & 50 & 50 & 0 & 0 & $327 \pm 5$ & 0.220 & $-11.6 \pm 0.3$ \\
\hline PrC 0.5 LS75 & 6 & 100 & 0 & 0 & 0.5 & 0 & $88 \pm 1$ & 0.250 & $-22 \pm 1$ \\
\hline PrT 0.5 LS75 & 7 & 0 & 100 & 0 & 0.5 & 0 & $415 \pm 19$ & 0.560 & $-18.9 \pm 0.2$ \\
\hline PrL 0.5 LS75 & 8 & 0 & 0 & 100 & 0.5 & 0 & $661 \pm 14$ & 0.440 & $-28.3 \pm 0.3$ \\
\hline PrTC 0.5 LS75 & 9 & 50 & 50 & 0 & 0.5 & 0 & $53.4 \pm 0.3$ & 0.190 & $-21.2 \pm 0.4$ \\
\hline PrTL 0.5 LS75 & 10 & 0 & 50 & 50 & 0.5 & 0 & $257 \pm 1$ & 0.190 & $-25.4 \pm 0.3$ \\
\hline PrC 1 LS75 & 11 & 100 & 0 & 0 & 1 & 0 & $80 \pm 1$ & 0.360 & $-35.97 \pm 0.1$ \\
\hline PrT 1 LS75 & 12 & 0 & 100 & 0 & 1 & 0 & $291 \pm 1$ & 0.400 & $-31 \pm 1$ \\
\hline PrL 1 LS75 & 13 & 0 & 0 & 100 & 1 & 0 & $182 \pm 5$ & 0.510 & $-32 \pm 1$ \\
\hline PrTC 1 LS75 & 14 & 50 & 50 & 0 & 1 & 0 & $64 \pm 1$ & 0.460 & $-20 \pm 2$ \\
\hline PrTL 1 LS75 & 15 & 0 & 50 & 50 & 1 & 0 & $65 \pm 1$ & 0.280 & $-22 \pm 1$ \\
\hline PrC 0.5 LSPC3 & 16 & 100 & 0 & 0 & 0 & 0.5 & $331 \pm 9$ & 0.330 & $-15 \pm 1$ \\
\hline PrT 0.5 LSPC3 & 17 & 0 & 100 & 0 & 0 & 0.5 & $364 \pm 12$ & 0.580 & $-17.1 \pm 0.5$ \\
\hline PrL 0.5 LSPC3 & 18 & 0 & 0 & 100 & 0 & 0.5 & $1684 \pm 108$ & 0.560 & $-14.8 \pm 0.5$ \\
\hline PrTC 0.5 LSPC3 & 19 & 50 & 50 & 0 & 0 & 0.5 & $73 \pm 1$ & 0.150 & $-12.1 \pm 0.2$ \\
\hline PrTL 0.5 LSPC3 & 20 & 0 & 50 & 50 & 0 & 0.5 & $319 \pm 4$ & 0.240 & $-13.0 \pm 0.2$ \\
\hline PrC 1 LSPC 3 & 21 & 100 & 0 & 0 & 0 & 1 & $442 \pm 10$ & 0.260 & $-13 \pm 1$ \\
\hline PrT 1 LSPC3 & 22 & 0 & 100 & 0 & 0 & 1 & $234 \pm 3$ & 0.400 & $-19 \pm 1$ \\
\hline PrL 1 LSPC3 & 23 & 0 & 0 & 100 & 0 & 1 & $1090 \pm 73$ & 0.490 & $-16 \pm 1$ \\
\hline PrTC 1 LSPC3 & 24 & 50 & 50 & 0 & 0 & 1 & $436 \pm 8$ & 0.680 & $-12 \pm 1$ \\
\hline PrTL 1 LSPC3 & 25 & 0 & 50 & 50 & 0 & 1 & $77 \pm 1$ & 0.240 & $-7.2 \pm 3$ \\
\hline
\end{tabular}

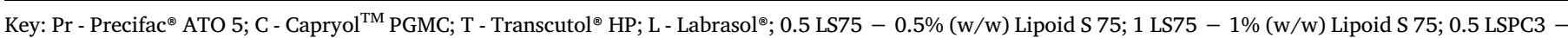
$0.5 \%(w / w)$ Lipoid S PC 3; 1 LSPC3 - 1\% (w/w) Lipoid S PC 3; PS - particle size; PI - polydispersity index; ZP - zeta potential. 


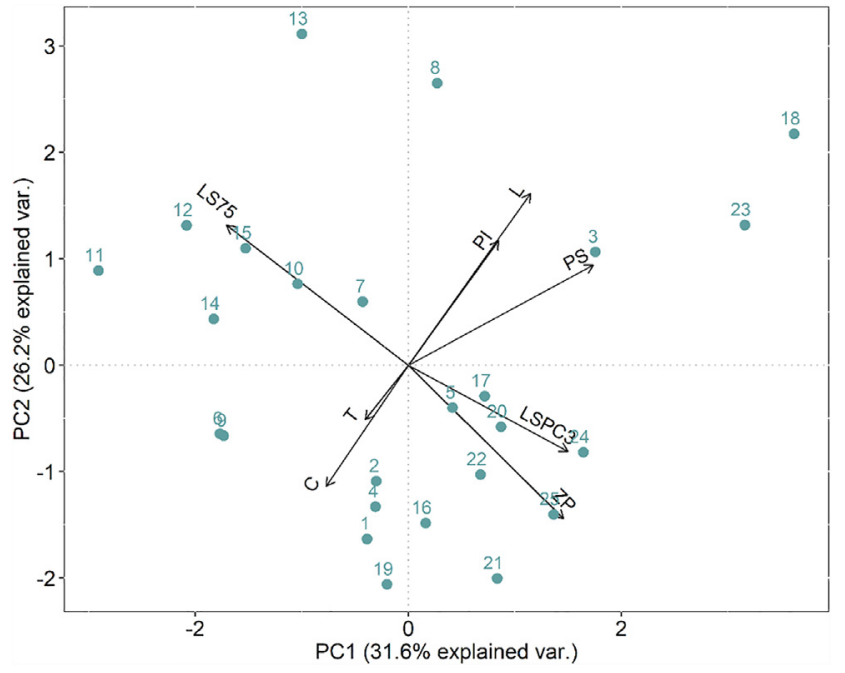

Fig. 10. Biplot representation of the produced NLCs and the eight considered variables, depicted in Table 4, on the first two principal components (PC1 and PC2). Discrimination among NLC formulations on the first two principal components is evident, recovering $57.8 \%$ of total variance. Key: C - Capryol ${ }^{\mathrm{TM}}$ PGMC; T - Transcutol ${ }^{\circledast}$ HP; L - Labrasol ${ }^{\oplus}$; LS75 - Lipoid S 75; LSPC3 - Lipoid S PC 3; PS - particle size; PI - polydispersity index; ZP - zeta potential.

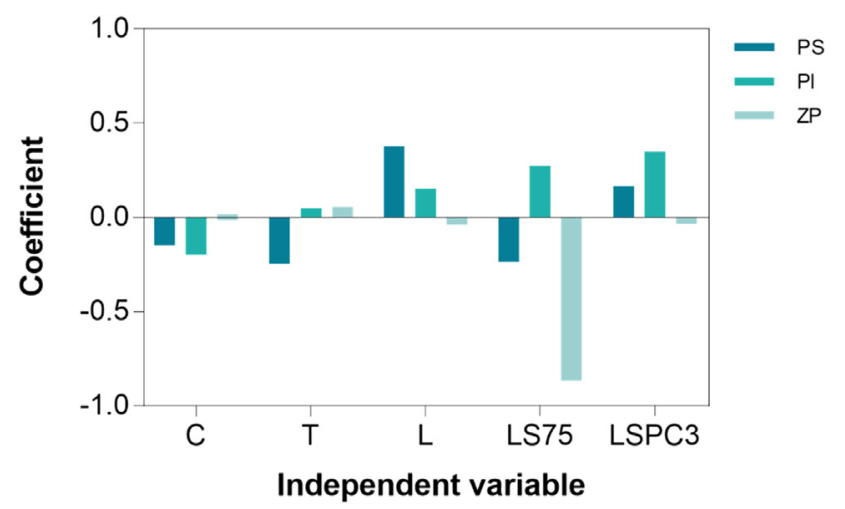

Fig. 11. Regression coefficients for PS, PI and ZP obtained from PLS applied to the NLCs depicted in Table 4. Key: C - Capryol ${ }^{\text {TM }}$ PGMC; T - Transcutol ${ }^{\circledast} \mathrm{HP}$; L Labrasol ${ }^{\circledR}$; LS75 - Lipoid S 75; LSPC3 - Lipoid S PC 3. Model was cross-validated (RMSEP, root mean square error for prediction) using 10 random segments (Tables S1 and S2, Supplementary Material).

\subsubsection{What DoE tells us?}

Establishing the right experimental set-up to address the intended purpose, i. e., to screen or optimize formulation/process parameters is not a random, but rather a timely planned procedure that must deserve particular attention. Full factorial designs, in particular those involving quadratic terms, provide a complete description of the process behavior, being useful to find out the optimal levels of the most important factors (Dejaegher and Vander Heyden, 2011). On the contrary, Taguchi designs can be used to assess the main effect of several factors with a relatively low number of experimental runs, presenting some time and cost associated advantages over full factorial designs. They use crossed inner and outer arrays to explore control factors in the presence of noise, thus adding a new dimension to conventional experimental design, playing an important role in producing high-quality products at subsequently low cost (Davis and John, 2018). Accordingly, $3^{\mathrm{k}}$ and $2^{\mathrm{k}}$ full factorial designs were applied, respectively, for composition optimization and method interchangeability assessment, while Taguchi design was employed for the development of biomimeting usNLCs.

$3^{\mathrm{k}}$ full factorial design

The most suitable NLC components for DoE were extracted from
PCA and PLS results. Although PLS describes how the concentration of each NLC component influences the studied responses, DoE ranks the impact of the composition on PS, PI, ZP, EE and DL.

Specifically, the effect of the concentration of T80 and composition of the lipid phase on PS, PI and ZP, as well as on EE and DL of both ATO and CUR, was investigated by a 9-run, two-factor and three-level response surface methodology. As previously described, ATO and CUR coencapsulated NLCs were prepared from P (solid lipid), T and L (liquid lipids) blends at different solid:liquid lipid ratios of 25:75, 50:50 and 75:25 (factor 2), and three different concentrations of aqueous surfactant T80 were considered, 1,3 and $5 \%(\mathrm{w} / \mathrm{w})$ (factor 1$)$. Both factors were selected due to their importance in the NLCs performance. In order to a priori minimize the source of variability from the production method, the homogenization and ultrasonication time, sample positioning and temperature were kept constant. The response results obtained for NLCs prepared according to the factorial planning are depicted in Fig. 12. According to the two-way ANOVA, statistically significant differences were observed for PS when the independent variables assume the three levels, except for NLCs with 1 and $5 \%(w / w)$ of T80 with a solid:liquid lipid ratio of 50:50 (p > 0.05). Significant differences were also identified for EE of ATO between NLCs with $1 \%$ $(\mathrm{w} / \mathrm{w})$ of T80 and solid:liquid lipid ratios of 25:75 and 50:50, as well as when the T80 concentration was varied $(1,3$ and $5 \%, w / w)$ on formulations with a solid:liquid lipid ratio of 25:75 ( $p<0.05)$. No significant differences $(\mathrm{p}>0.05)$ were observed for the remaining dependent variables (PI, ZP, DL of ATO, and EE and DL of CUR).

The regression coefficients obtained for each dependent variable are summarized in Table 4. The one-way ANOVA (Table S4, Supplementary Material) confirmed that the generated regression models are significant, with the exception of the models defined to describe the DL of ATO and CUR ( $p>0.05)$.

Response and contour surface representations were constructed to elucidate the effect of the selected factors on the dependent variables (Fig. 13 for PS, PI and ZP; Fig. 14 for EE of ATO and CUR). The predictability of the response surface models was validated resorting to a NLC composed of 5\% T80 and a solid:liquid lipid ratio of 70:30 (level $[+1,+0.8]$ ) and is presented in Table S3, Supplementary Material. Concentration of T80 has a significant impact on PS, suggesting that the nanosystem properties are mainly governed by the amount of aqueous surfactant. The negative sign of $\beta_{1}$ indicates that smaller PS values are obtained by increasing the T80 concentration. The stabilization of NLCs with higher amount of aqueous surfactant has been previously described (Vitorino et al., 2013; Mendes et al., 2016). The same effect was obtained considering the solid:liquid lipid ratio, but in a lesser extent, where a higher amount of solid lipid prompted a smaller PS. Note that, $\beta_{2}$ was not considered statistically significant $(p>0.05)$. The interaction between the factors has a higher impact than the isolated effect and the positive sign of $\beta_{12}$, as well as the interaction plots displayed in Fig. 15, indicated that, for higher T80 concentration and amount of solid lipid, the interaction favors PS. Additionally, it was observed that PI is also affected by the T80 concentration and the solid:liquid lipid ratio. Higher levels of these factors induced more homogeneous NLCs, and the interaction was considered negligible and not considered statistically significant ( $\mathrm{p}>0.05$ ). ZP is significantly influenced by the T80 concentration, where the positive sign of $\beta_{1}$ denotes that the high level of this factor contributes to less negative ZP values, and, consequently, less stable NLCs. However, no effect was found for the solid:liquid lipid ratio on ZP. The T80 concentration was also the factor with the most pronounced effect on the EE of ATO, followed by the composition of lipid phase. The positive and negative signs of $\beta_{1}$ and $\beta_{2}$, respectively, mean that an increase in the EE might be achieved considering a high T80 concentration and a low amount of solid lipid. Moreover, the negative sign of the $\beta_{12}$, and also the trend observed in the interaction profiles of Fig. 15 indicate that the interaction reduces the positive effect of factor 1 on the EE, decreasing it (for a higher T80 concentration and amount of solid lipid). However, the EE of CUR was 

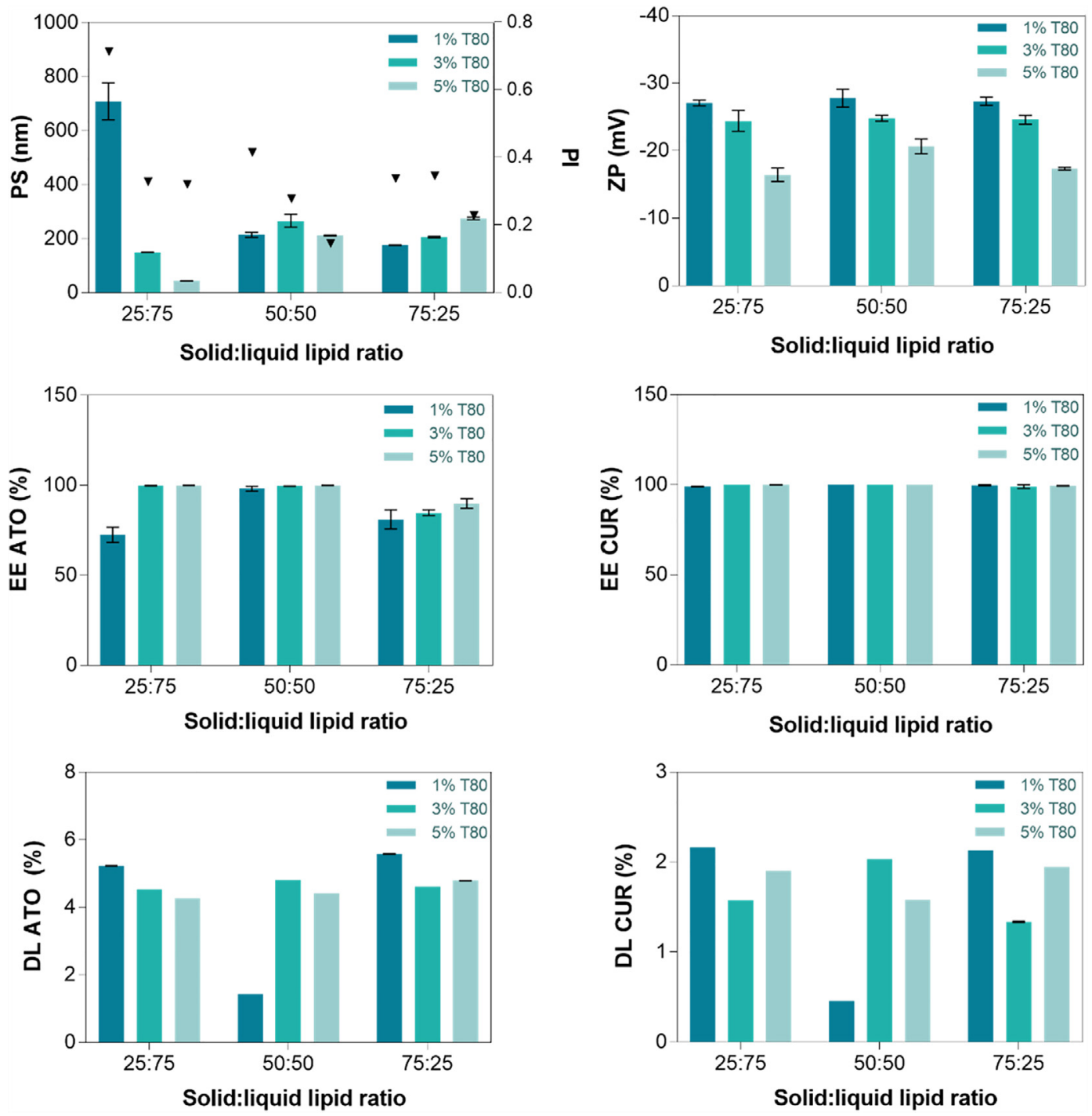

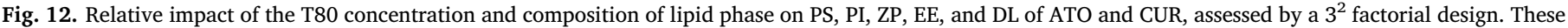
NLCs were produced by ultrasonication. Responses were evaluated in triplicate.

Table 4

Regression coefficients obtained from the $3^{2}$ factorial design, for each dependent variable, PS, PI, ZP and EE of ATO and CUR (*p > 0.05).

\begin{tabular}{|c|c|c|c|c|c|c|}
\hline \multirow[t]{2}{*}{ Response } & \multicolumn{6}{|c|}{ Coefficient } \\
\hline & $\boldsymbol{\beta}_{0}$ & $B_{1}$ & $\boldsymbol{B}_{2}$ & $B_{12}$ & $\boldsymbol{\beta}_{11}$ & $B_{22}$ \\
\hline PS & 157.29 & -119.26 & $-39.91 *$ & 192.40 & $39.93^{*}$ & 75.01 \\
\hline PI & 0.27 & -0.08 & -0.09 & $0.04^{*}$ & 0.09 & $0.06 *$ \\
\hline ZP & -24.48 & 4.45 & $0.48^{*}$ & 0.87 & 1.66 & -0.16 \\
\hline EE ATO & 102.20 & 6.35 & -2.75 & -4.66 & $-4.45^{*}$ & -11.20 \\
\hline EE CUR & 99.98 & $0.08 *$ & $-0.15^{*}$ & -0.29 & $0.03^{*}$ & -0.43 \\
\hline
\end{tabular}

not significantly influenced by the two factors.

In order to select the optimal formulation composition, the five CQAs were simultaneously analysed with regions limiting each CQA (Fig. 16), aiming at minimizing PS and PI and maximizing EE and $|\mathrm{ZP}|$. The white area, designated as Design Space, defines a limited region in which the formulations gather the desired properties. To sum-up, the optimal conditions to assemble the best performance of the NLCs, including small and homogenous usNLCs, with high stability and drug encapsulation, and meeting the suitable CQAs for intravenous administration, are achieved using a higher T80 concentration $(+1)$, and a lower amount of solid lipid, i.e. a solid:liquid lipid ratio of 25:75 (-1). $2^{\mathrm{k}}$ full factorial design

The influence of the T80 concentration and the production method on PS, PI and ZP was further assessed by a 4-run, two-factor and twolevel full factorial design. This second DoE is essentially directed to the study of method interchangeability, aiming at a potential scaling-up process by using high pressure homogenization. The considered unloaded NLCs were prepared from P (solid lipid), T and L (liquid lipids) blends with a solid:liquid lipid ratio of 25:75, by two different methods, namely US and HPH (factor 2). Different concentrations of T80 were also considered ( 1 and $5 \%, \mathrm{w} / \mathrm{w}$ ) (factor 1 ). Note that the production method variables were kept the same. The response results obtained for NLCs prepared according to the factorial planning are depicted in Fig. 17. The one-way ANOVA (Table S5, Supplementary Material) confirmed that the generated regression models are significant ( $\mathrm{p}<0.05)$. Regarding the two-way ANOVA, statistically significant differences $(\mathrm{p}<0.05)$ for PS and ZP were observed between NLCs developed with different T80 concentrations and using different production methods, except among NLCs with $5 \%(w / w)$ of T80 produced either by US or HPH ( $p>0.05$ ). For PI, no significant differences were found ( $p>0.05$ ). Table 5 presents the regression coefficients obtained for each dependent variable. The T80 concentration significantly impacts PS, with higher T80 concentrations providing the smallest NLCs, 


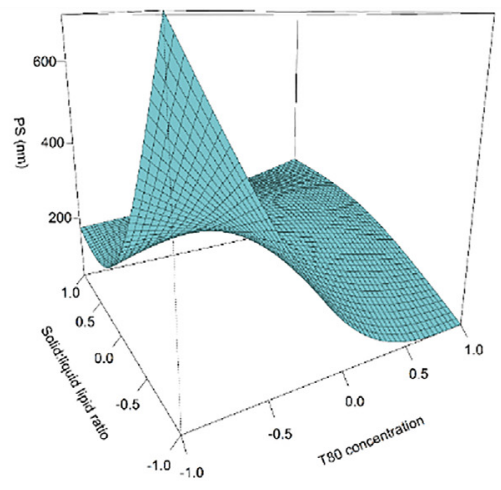

PS (nm)

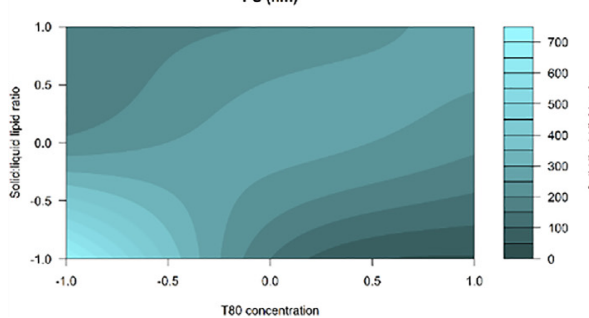

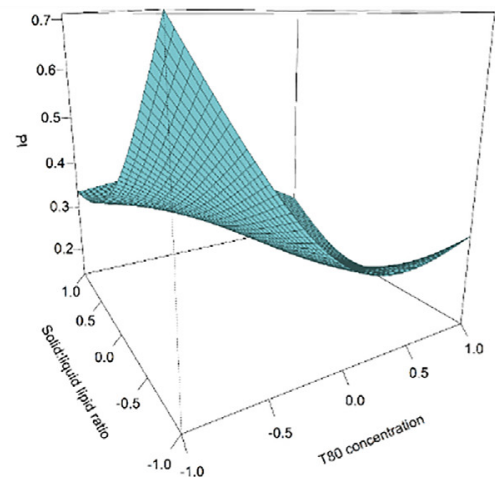

PI

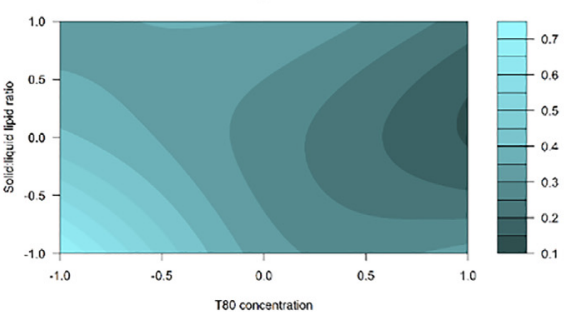

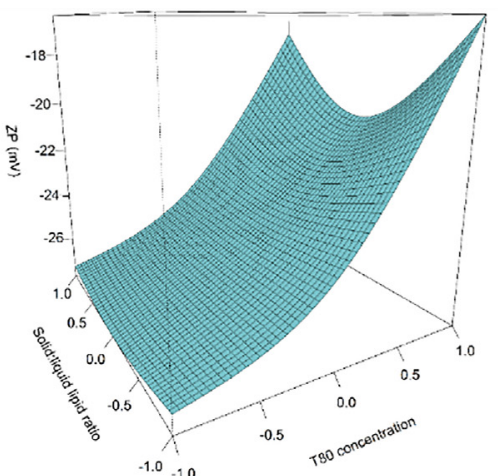

$\mathrm{ZP}(\mathrm{mV})$

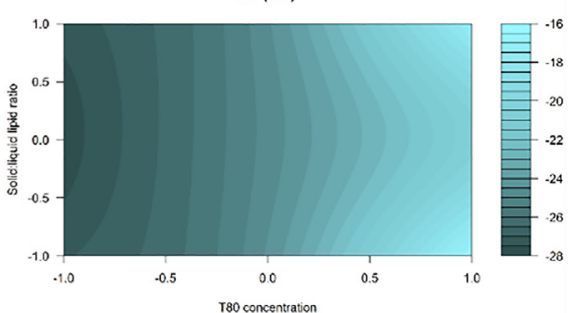

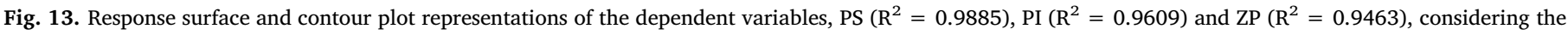
concentration of T80 and the solid:liquid lipid ratio. The desired conditions rely on the minimization of PS and PI, and maximization of $|\mathrm{ZP}|$.

as previously observed in the $3^{2}$ factorial planning. Also, the type of production method plays an important role on PS, although in a lesser extent. ZP increased when a higher T80 concentration was considered, and were more negative when the HPH method was used. The interaction between the two factors was also evaluated for each of the dependent variables and significant interactions were identified for PS

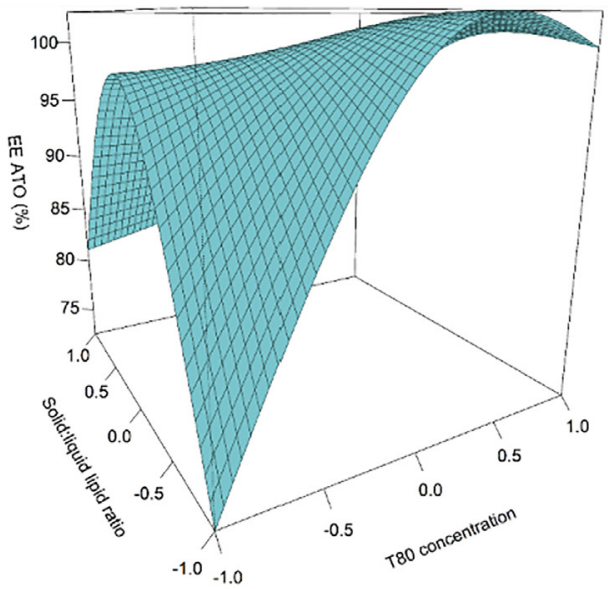

EE ATO (\%)

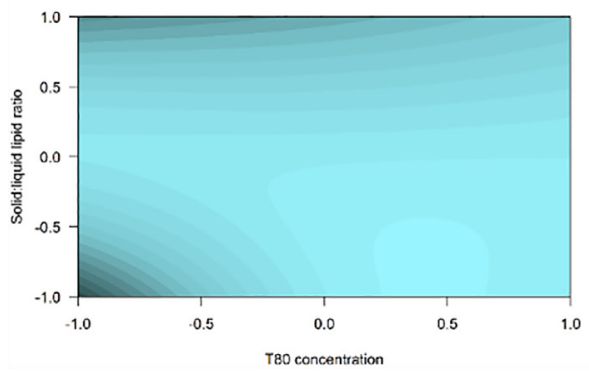

and ZP ( $\mathrm{p}<0.05)$. The negative and positive signs of $\beta_{12}$ for PS and $\mathrm{ZP}$, respectively, as well as the interaction plots displayed in Fig. 18, suggest that the interaction between high levels of T80 and the production method (i.e. HPH) reinforces the negative effect of factor 1 on PS and the positive influence of factor 1 on ZP. Also, the interaction between factors decreases the positive effect of factor 2 on PS and the
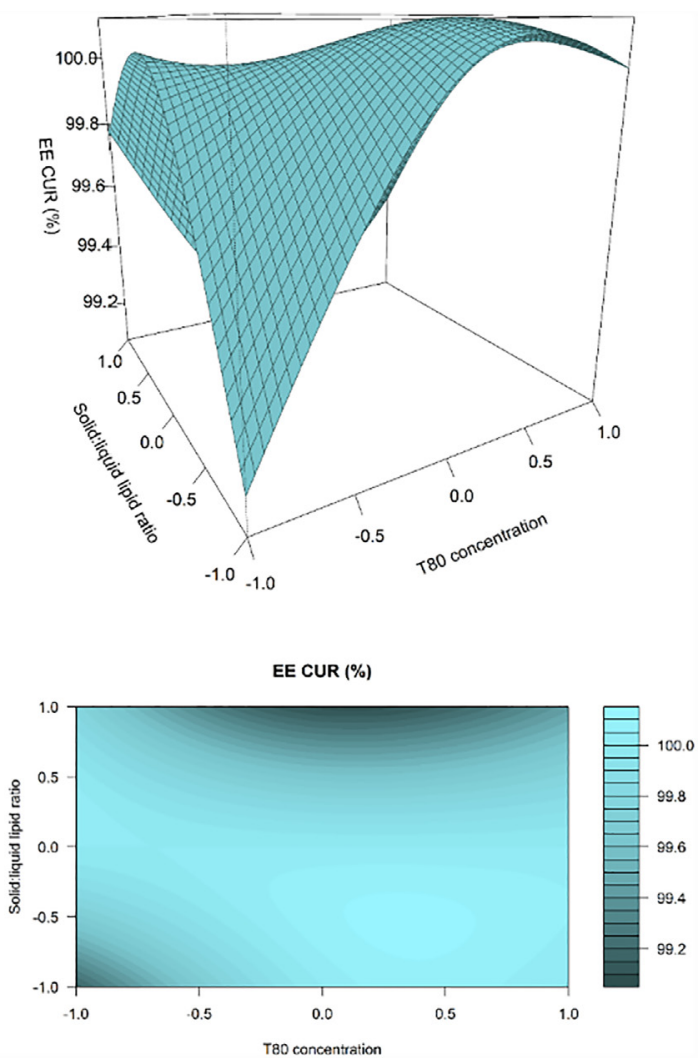

Fig. 14. Response surface and contour plot representations of the dependent variables, EE of ATO $\left(R^{2}=0.9562\right)$ and CUR $\left(R^{2}=0.6066\right)$ considering the concentration of T80 and the solid:liquid lipid ratio. The desired conditions rely on the maximization of EE. 

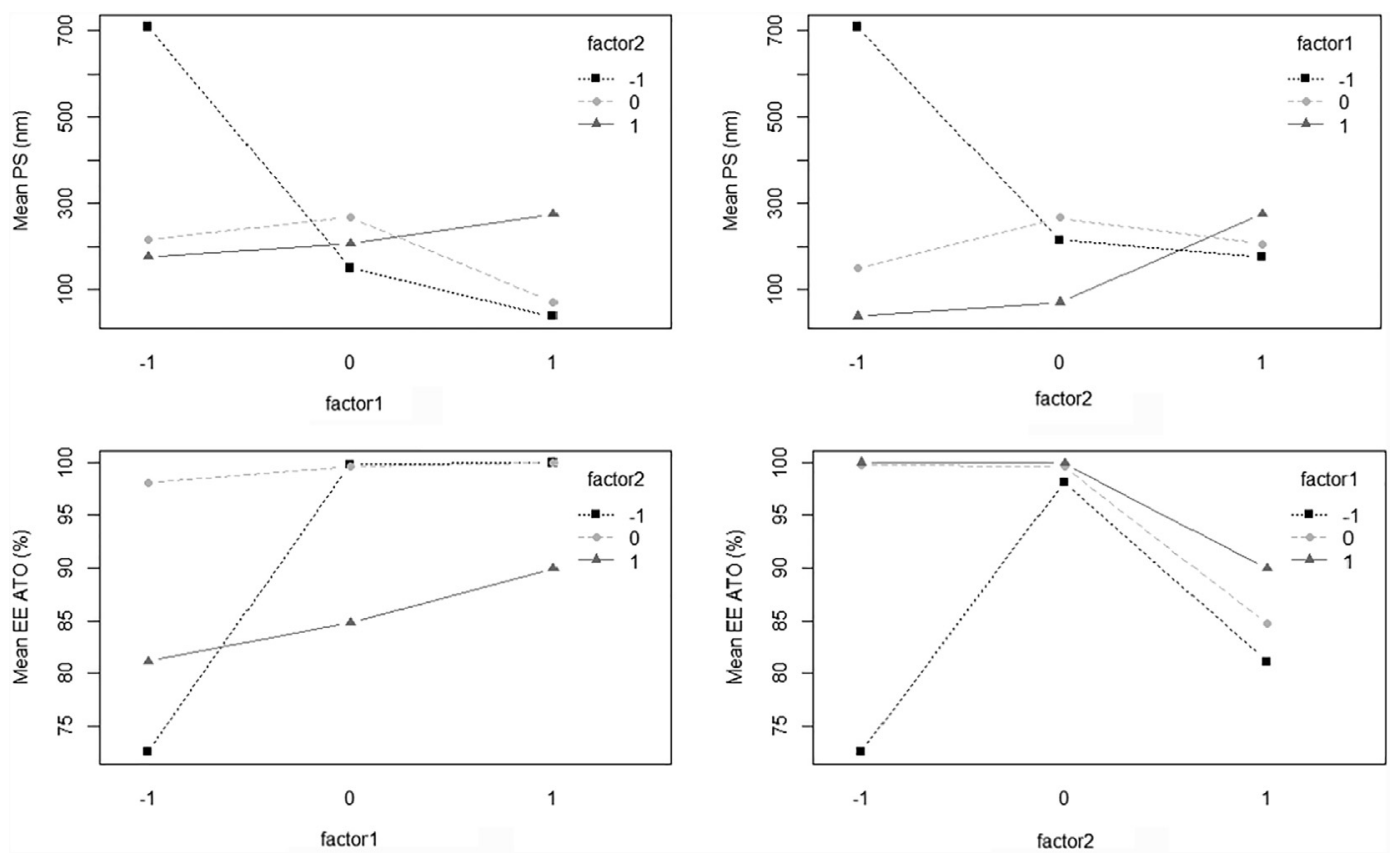

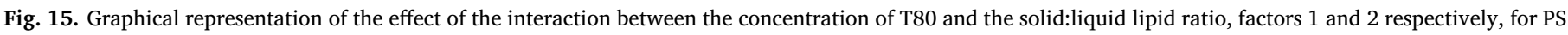
and EE of ATO, within the range of the experiment.

negative influence of factor 2 on ZP, leading to smaller and less negatively charged NLCs.

To sum-up, at a higher concentration of T80 concentration, irrespective of the production method, usNLCs with similar PS, PI and ZP are obtained, indicating that the production methods are reproducible and can be interchanged. This allows taking advantage of the easily upscalable feature of HPH to be employed in the industrial environment, while the scalability of US method is still questionable (Tapeinos et al.,
2017; Ganesan and Narayanasamy, 2017).

After the optimization step, the risk assessment matrix was updated according to the reduced level of risk inspected with the DoE application (Table 6). The solid:liquid lipid ratio still displays a high impact on PS, but the risk level assigned to PI and ZP, and to EE and DL was reduced to low and medium risk, respective. Also, the risk levels of surfactant concentration and production method assigned to PI were reduced to low levels.

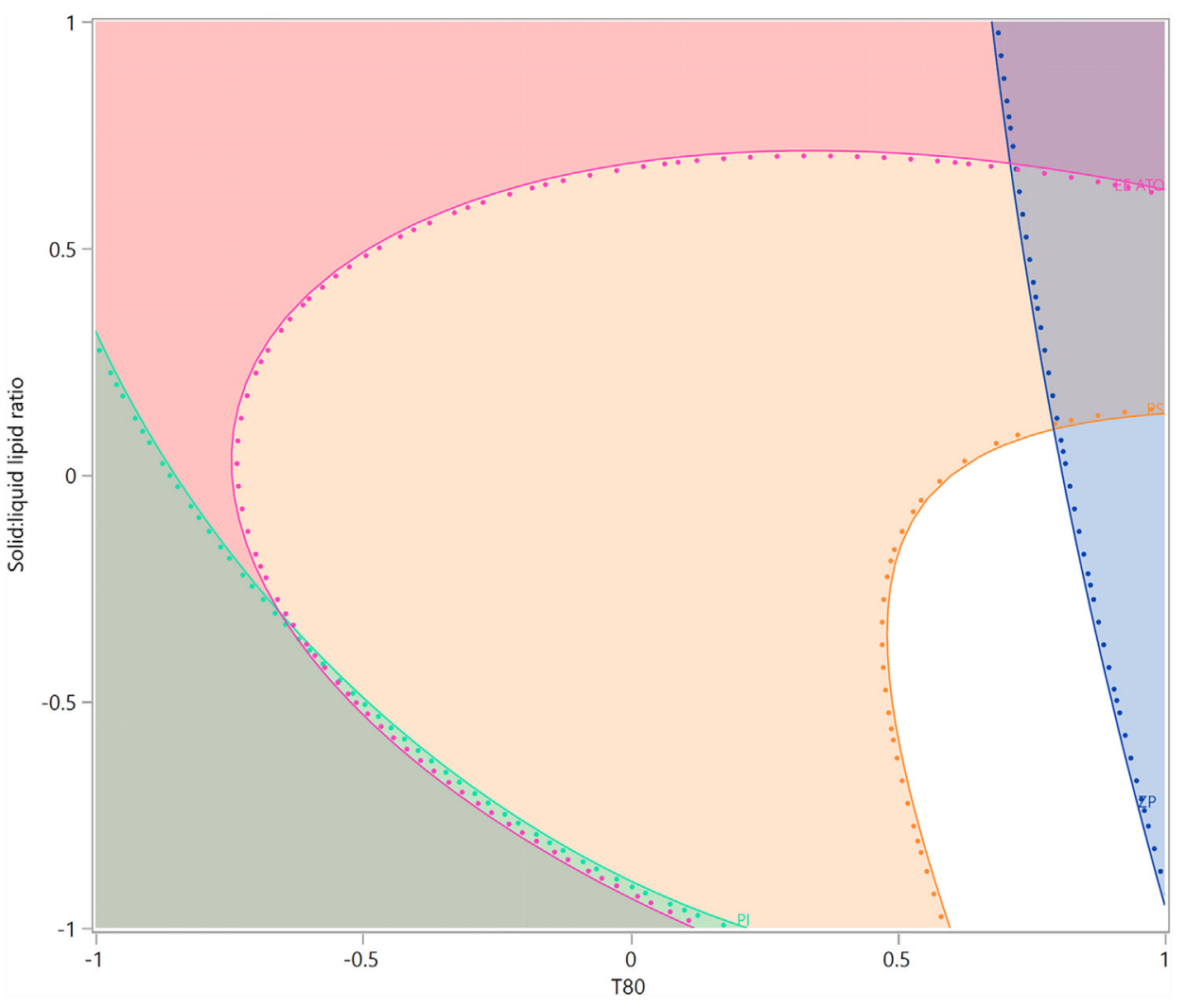

Fig. 16. Definition of the Design Space, the region that provides the optimal NLC composition, in terms of solid:liquid lipid ratio and surfactant content. To determine this region, PS and PI were limited to a maximum of $100 \mathrm{~nm}$ and 0.4 , respectively, while ZP, EE and DL were limited to a minimum value of $-20 \mathrm{mV}$ and $95 \%$, respectively (the dots indicate the direction to which the magnitude of the CQA increases). The model was validated with the use of an additional NLC (Table S3, Supplementary Material). 

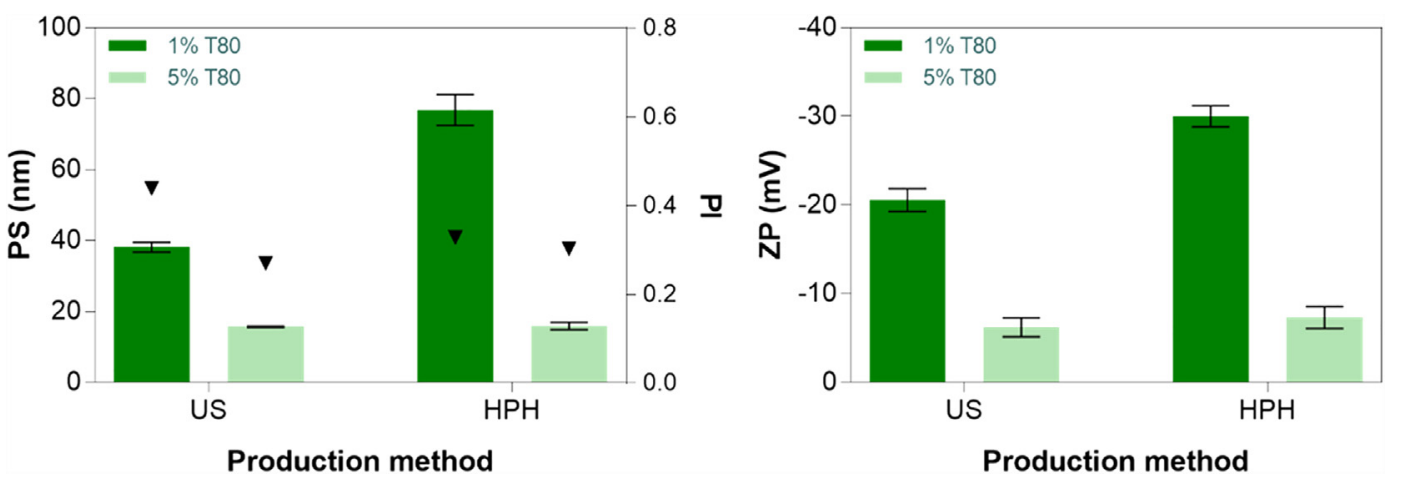

Fig. 17. Influence of the concentration of T80 and production method, US and HPH, on PS, PI and ZP, evaluated by a $2^{2}$ factorial design.

Table 5

Regression coefficients obtained from the $2^{2}$ factorial design, for each dependent variable, PS, PI and ZP (*p > 0.05).

\begin{tabular}{|c|c|c|c|c|}
\hline \multirow[t]{2}{*}{ Response } & \multicolumn{4}{|c|}{ Coefficient } \\
\hline & $B_{0}$ & $\beta_{1}$ & $\beta_{2}$ & $B_{12}$ \\
\hline PS & 46.68 & -20.85 & 9.72 & -9.62 \\
\hline PI & 0.34 & -0.05 & $-0.02^{*}$ & 0.04 \\
\hline $\mathrm{ZP}$ & -16.03 & 9.27 & -2.64 & 2.09 \\
\hline
\end{tabular}

\subsection{Characterizing nanocarriers}

Solid lipid nanoparticles, including NLCs, are characterized by the presence of a solid lipid matrix. The introduction of a liquid lipid may modify the physicochemical properties of the carrier. Following the results of the DOE, and to infer the impact of the composition of the lipid matrix on the physicochemical properties of the NLCs, a further characterization throughout time and drug release was conducted.

\subsubsection{Stability}

The physicochemical stability of the NLCs containing different
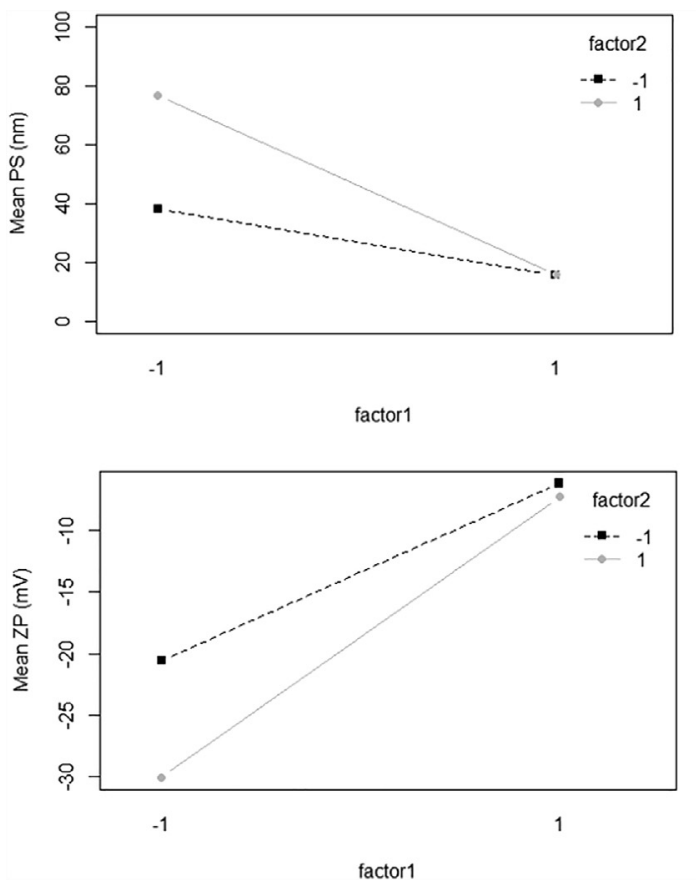

solid:liquid lipid ratios (25:75, 50:50 and 75:25) was assessed through the evaluation of PS, PI and ZP at predefined days after production, with the results being presented in Fig. 19. It is possible to observe that, although the PS slowly increases in the NLC with a solid:liquid lipid ratio of 25:75, the PI decreases over time. On the other hand, NLCs with solid:liquid lipid ratios of 50:50 and 75:25 are less stable, expressing more pronounced variability on PS and PI over the 180 days evaluation and presenting both PS and PI higher than the corresponding parameters in the 25:75 ratio formulation. Regarding the ZP, no relevant variations were identified over time.

\subsubsection{In vitro release behavior}

Release profiles of NLCs displayed in Fig. 20 exhibit a sustained drug release throughout the study for CUR and ATO, when compared to a control solution containing both compounds ( $p<0.05$ ). NLCs containing $75 \%$ of solid lipid present the lowest release rate for CUR, which suggests the compound is entrapped within the solid lipid. An ANOVA was conducted, and no significant differences were found between NLCs containing 25 and $50 \%$ of solid lipid in their composition ( $\mathrm{p}>0.05$ ). All NLCs seem to improve CUR stability at $\mathrm{pH}=7.4$, as the control solution shows extensive CUR degradation following $9 \mathrm{~h}$. When compared to CUR, a higher amount of ATO is released within the same
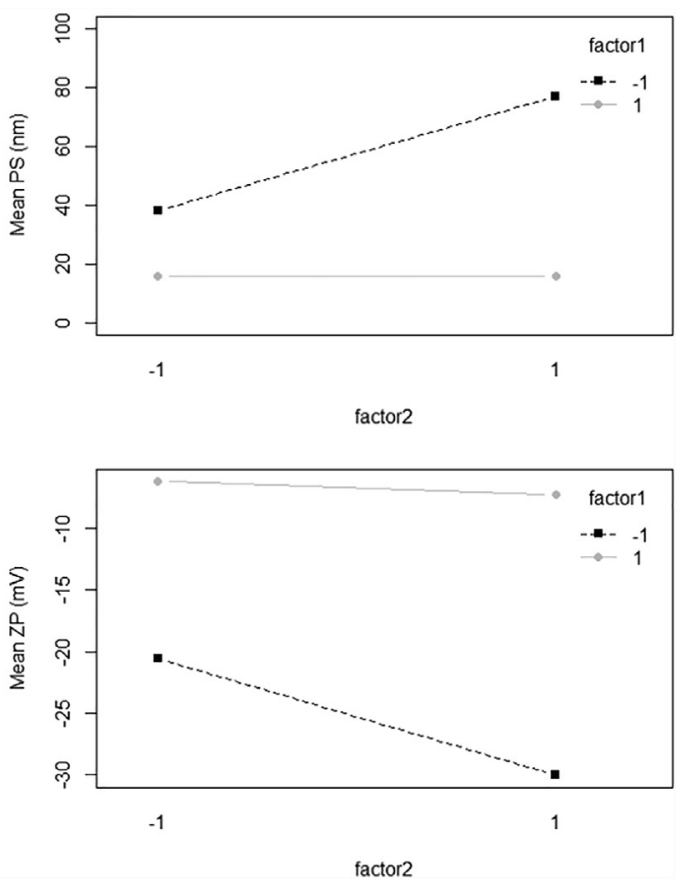

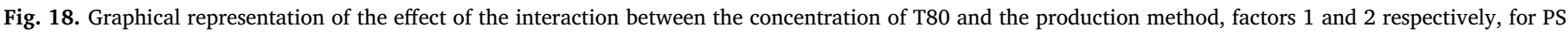
and $\mathrm{ZP}$ within the range of the experiment. 
Table 6

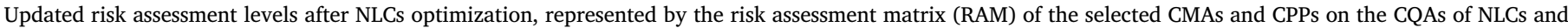
qualitatively ranked as low-, medium- or high-risk.

\begin{tabular}{|c|c|c|c|c|c|c|c|c|}
\hline \multirow[t]{2}{*}{ CQAs } & \multicolumn{7}{|l|}{ CMAs } & \multirow{2}{*}{$\begin{array}{l}\text { CPPs } \\
\begin{array}{l}\text { Homogenization } \\
\text { method type }\end{array}\end{array}$} \\
\hline & $\begin{array}{l}\text { Type of } \\
\text { lipid }\end{array}$ & $\begin{array}{l}\text { Lipid } \\
\text { concentration }\end{array}$ & $\begin{array}{l}\text { Solid:liquid lipid } \\
\text { ratio }\end{array}$ & $\begin{array}{l}\text { Type of } \\
\text { surfactant }\end{array}$ & $\begin{array}{l}\text { Surfactant } \\
\text { concentration }\end{array}$ & $\begin{array}{l}\text { Drug } \\
\text { concentration }\end{array}$ & $\begin{array}{l}\text { Drug } \\
\text { solubility }\end{array}$ & \\
\hline PS & Medium & Medium & High & Medium & High & Medium & Low & High \\
\hline PI & Low & Low & Low & Medium & Low & Medium & Low & Low \\
\hline ZP & Medium & Medium & Low & High & High & Medium & Low & Medium \\
\hline EE & Medium & High & Medium & Medium & Medium & High & High & Low \\
\hline DL & Medium & High & Medium & Low & Medium & High & High & Low \\
\hline Drug release & Medium & Medium & High & Medium & High & Medium & High & Low \\
\hline
\end{tabular}

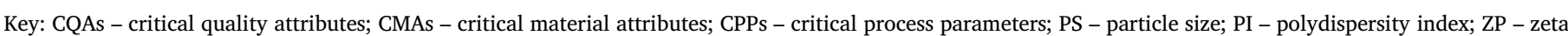
potential; EE - entrapment efficiency; DL - drug loading.
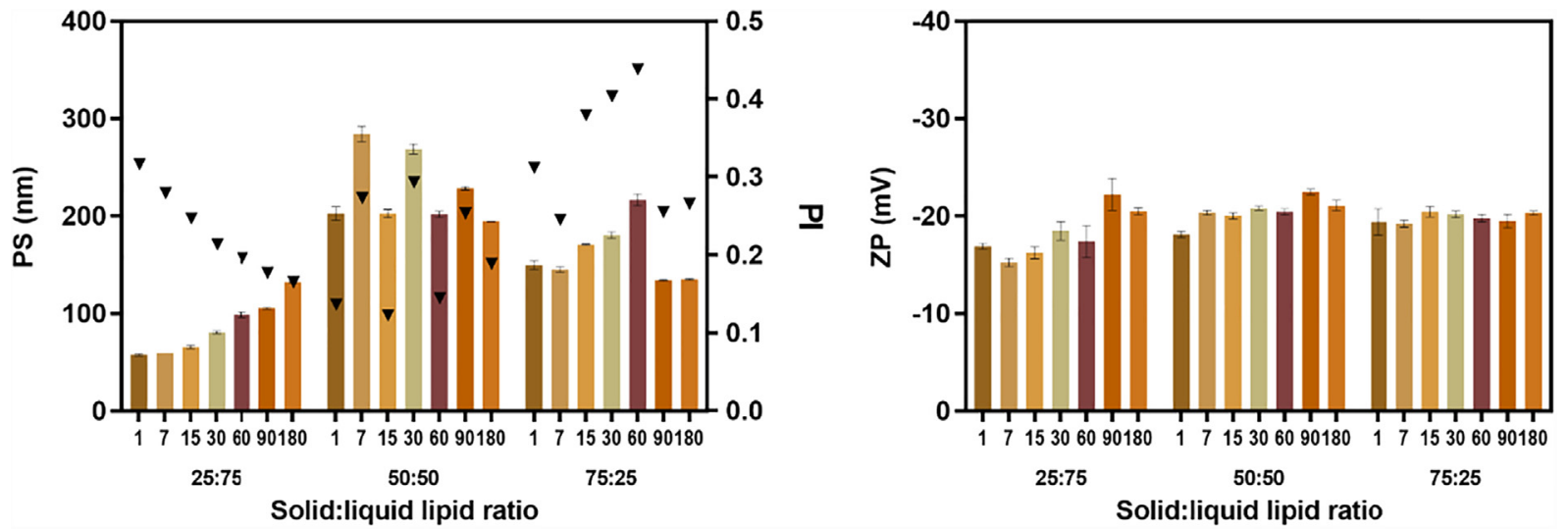

Fig. 19. Monitoring of the physicochemical stability of NLCs through the evaluation of PS, PI and ZP over time.

time-period, indicating a less stable behavior of this drug in the system. In fact, ATO is more soluble in the aqueous phase of the formulation and in the release medium, by a 20 and 40 -fold factor, respectively. Within the first $3 \mathrm{~h}$ of the study, the release behavior of free and loaded ATO through the dialysis membrane is similar. Additionally, at $48 \mathrm{~h}$, $90-100 \%$ of the drug was released to the medium. The controlled release of ATO appears to be independent of the solid:liquid lipid ratio, considering no significant differences were identified between the NLCs ( $\mathrm{p}>0.05)$.

In order to compare the release profiles and evaluate the release kinetics, different methods were applied. Regarding the first approach where a model independent method was used, the similarity factor $f_{2}$ was calculated using each one of the release profiles as reference, for ATO and CUR separately (Table 7). As mentioned above, $f_{2}$ values greater than 50 suggest that the two profiles are similar. Considering the control solution as reference, the $f_{2}$ values for ATO and CUR were
Table 7

Similarity factors $f_{2}$ obtained after comparing the release profiles of the NLCs and control solution for ATO and CUR ( $\mathrm{n}=11$; for *, $\mathrm{n}=9$ ).

\begin{tabular}{lllllllll}
\hline$f_{2}$ & ATO & \multicolumn{8}{c}{ CUR } \\
\cline { 2 - 9 } & $25: 75$ & $50: 50$ & $75: 25$ & Control & $25: 75$ & $50: 50$ & $75: 25$ & Control \\
\hline $25: 75$ & 100 & - & - & - & 100 & - & - & - \\
$50: 50$ & 57.01 & 100 & - & & 97.19 & 100 & - & - \\
$75: 25$ & 60.39 & 77.91 & 100 & - & 74.99 & 75.26 & 100 & - \\
Control & $34.76^{*}$ & $40.99^{*}$ & $42.16^{*}$ & $100^{*}$ & 48.82 & 49.46 & 46.64 & 100 \\
\hline
\end{tabular}

found to be lower than 50, meaning that no similarity is evidenced between control profiles and the remaining ones. On the other hand, after crossing all NLCs with each other, it was found that there is similarity among them, since the $f_{2}$ values are above 50 .
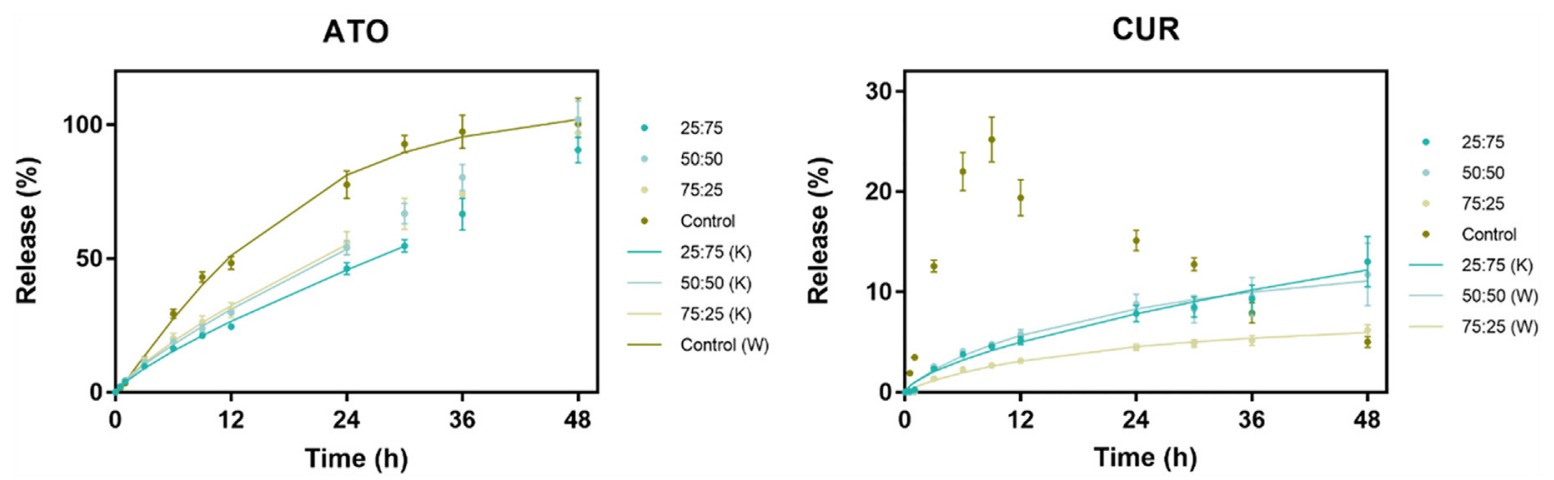

Fig. 20. Release profiles of NLCs for ATO and CUR, according to different ratios of solid:liquid lipids and control, before (dots) and after (solid lines) the application of the best fitting model, Korsmeyer-Peppas (K) or Weibull (W). Results are related to predetermined intervals and are expressed as mean \pm SEM $(6 \leq \mathrm{n} \leq 12)$. 
Table 8

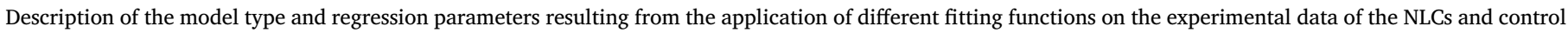
solution for ATO and CUR.

\begin{tabular}{|c|c|c|c|c|c|c|}
\hline Formulation & Drug & Function & $\mathrm{c}_{1}$ & $\mathrm{c}_{2}$ & $\mathrm{c}_{3}$ & $r^{2}$ \\
\hline \multirow[t]{10}{*}{$25: 75$} & \multirow[t]{5}{*}{ Ато } & Zero-order $^{\mathrm{a}}$ & $1.89 \pm 0.04$ & - & - & 0.9964 \\
\hline & & First-order ${ }^{\mathrm{b}}$ & $357 \pm 216$ & $0.006 \pm 0.004$ & - & 0.9953 \\
\hline & & Higuchi $^{\mathrm{c}}$ & $8.6 \pm 0.6$ & - & - & 0.9631 \\
\hline & & Weibull $^{\mathrm{d}}$ & $-*$ & -* & $-*$ & 0.9962 \\
\hline & & Korsmeyer-Peppas ${ }^{\mathrm{e}}$ & $3.7 \pm 0.3$ & $0.79 \pm 0.02$ & - & 0.9981 \\
\hline & \multirow[t]{5}{*}{ CUR } & Zero-order $^{\mathrm{a}}$ & $0.29 \pm 0.02$ & - & - & 0.9504 \\
\hline & & First-order ${ }^{\mathrm{b}}$ & $14 \pm 2$ & $0.04 \pm 0.01$ & - & 0.9685 \\
\hline & & Higuchi $^{\mathrm{c}}$ & $1.63 \pm 0.07$ & - & - & 0.9779 \\
\hline & & Weibull $^{\mathrm{d}}$ & $-*$ & $-*$ & $-*$ & 0.9831 \\
\hline & & Korsmeyer-Peppas ${ }^{\mathrm{e}}$ & $1.0 \pm 0.2$ & $0.64 \pm 0.05$ & - & 0.9832 \\
\hline \multirow{10}{*}{$50: 50$} & \multirow{5}{*}{ ATO } & Zero-order $^{\mathrm{a}}$ & $2.21 \pm 0.05$ & - & - & 0.9967 \\
\hline & & First-order ${ }^{\mathrm{b}}$ & $267 \pm 65$ & $0.010 \pm 0.003$ & - & 0.9977 \\
\hline & & Higuchi $^{\mathrm{c}}$ & $9.2 \pm 0.7$ & - & - & 0.9577 \\
\hline & & Weibull $^{\mathrm{d}}$ & $-*$ & $-*$ & $-*$ & 0.9831 \\
\hline & & Korsmeyer-Peppas ${ }^{\mathrm{e}}$ & $4.3 \pm 0.3$ & $0.79 \pm 0.03$ & - & 0.9980 \\
\hline & \multirow[t]{5}{*}{ CUR } & Zero-order $^{\mathrm{a}}$ & $0.28 \pm 0.02$ & - & - & 0.9038 \\
\hline & & First-order ${ }^{\mathrm{b}}$ & $11.3 \pm 0.9$ & $0.06 \pm 0.01$ & - & 0.9762 \\
\hline & & Higuchi $^{\mathrm{c}}$ & $1.63 \pm 0.06$ & - & - & 0.9797 \\
\hline & & Weibull $^{\mathrm{d}}$ & $15 \pm 6$ & $0.03 \pm 0.03$ & $0.8 \pm 0.2$ & 0.9824 \\
\hline & & Korsmeyer-Peppas ${ }^{\mathrm{e}}$ & $1.3 \pm 0.2$ & $0.56 \pm 0.05$ & - & 0.9774 \\
\hline \multirow[t]{10}{*}{$75: 25$} & \multirow[t]{5}{*}{ ATO } & Zero-order $^{\mathrm{a}}$ & $2.14 \pm 0.07$ & - & - & 0.9906 \\
\hline & & First-order ${ }^{\mathrm{b}}$ & $166 \pm 25$ & $0.017 \pm 0.004$ & - & 0.9963 \\
\hline & & Higuchi $^{\mathrm{c}}$ & $9.6 \pm 0.7$ & - & - & 0.9641 \\
\hline & & Weibull $^{\mathrm{d}}$ & $-*$ & $-*$ & $-*$ & 0.9891 \\
\hline & & Korsmeyer-Peppas ${ }^{\mathrm{e}}$ & $4.8 \pm 0.3$ & $0.77 \pm 0.03$ & - & 0.9979 \\
\hline & \multirow[t]{5}{*}{ CUR } & Zero-order ${ }^{\mathrm{a}}$ & $0.15 \pm 0.01$ & - & - & 0.9024 \\
\hline & & First-order ${ }^{\mathrm{b}}$ & $6.1 \pm 0.4$ & $0.062 \pm 0.009$ & - & 0.9848 \\
\hline & & Higuchi $^{\mathrm{c}}$ & $0.88 \pm 0.03$ & - & - & 0.9842 \\
\hline & & Weibull $^{\mathrm{d}}$ & $7 \pm 2$ & $0.04 \pm 0.02$ & $0.8 \pm 0.1$ & 0.9891 \\
\hline & & Korsmeyer-Peppas ${ }^{\mathrm{e}}$ & $0.7 \pm 0.1$ & $0.56 \pm 0.05$ & - & 0.9816 \\
\hline \multirow{10}{*}{ Control } & \multirow[t]{5}{*}{ ATO } & Zero-order $^{\mathrm{a}}$ & $2.7 \pm 0.2$ & - & - & 0.9159 \\
\hline & & First-order ${ }^{\mathrm{b}}$ & $115 \pm 5$ & $0.049 \pm 0.005$ & - & 0.9959 \\
\hline & & Higuchi $^{\mathrm{c}}$ & $12 \pm 1$ & - & - & 0.9359 \\
\hline & & Weibull $^{\mathrm{d}}$ & $107 \pm 5$ & $0.057 \pm 0.006$ & $1.13 \pm 0.09$ & 0.9968 \\
\hline & & Korsmeyer-Peppas ${ }^{\mathrm{e}}$ & $5 \pm 1$ & $0.9 \pm 0.1$ & - & 0.9854 \\
\hline & \multirow[t]{5}{*}{ CUR } & Zero-order ${ }^{\mathrm{a}}$ & $0.4 \pm 0.2$ & - & - & 0.0004 \\
\hline & & First-order ${ }^{\mathrm{b}}$ & $15 \pm 3$ & $0.6 \pm 0.6$ & - & 0.5210 \\
\hline & & Higuchi $^{\mathrm{c}}$ & $2.7 \pm 0.8$ & - & - & 0.0478 \\
\hline & & Weibull $^{\mathrm{d}}$ & $15 \pm 3$ & $0.5 \pm 0.4$ & $2 \pm 2$ & 0.5448 \\
\hline & & Korsmeyer-Peppas ${ }^{\mathrm{e}}$ & $10 \pm 5$ & $0.1 \pm 0.2$ & - & 0.2447 \\
\hline
\end{tabular}

${ }^{\mathrm{a}} \mathrm{c}_{1} t ;{ }^{\mathrm{b}} \mathrm{c}_{1}\left(1-\exp \left(-\mathrm{c}_{2} t\right)\right) ;{ }^{\mathrm{c}} \mathrm{c}_{1} \mathrm{t}^{0.5} ;{ }^{\mathrm{d}} \mathrm{c}_{1}\left(1-\exp \left(-\mathrm{c}_{2} \mathrm{t}^{\mathrm{c} 3}\right)\right) ;{ }^{\mathrm{e}} \mathrm{c}_{1} \mathrm{t}^{\mathrm{c} 2 ;} ;{ }^{*}$ Despite the relatively high $r^{2}$ values obtained, the uncertainty associated to the estimated parameters does not provide a good ATO or CUR release fitting.

Additionally, different model dependent methods were implemented to fit and describe the drug release profile. The regression parameters obtained for each mathematical function are depicted in Table 8, as well as the corresponding coefficients of determination, $r^{2}$, which reflect the goodness of fitting. Considering the $r^{2}$, it was found that the Korsmeyer-Peppas model provides the best fitting model for ATO in the three evaluated NLCs, as well as for CUR in the NLCs with a solid:liquid lipid ratio of 25:75. The $c_{2}$ parameter of the KorsmeyerPeppas equation, defined as the release exponent, aids at characterizing the drug release. According to the calculated values of the release exponent for the previously mentioned NLCs $\left(0.5<c_{2}<1\right)$, it is possible to conclude that their release mechanism follows a non-Fickian diffusion process (Cascone, 2017; Dash et al., 2010). Note that this semi-empirical model just considers the first $60 \%$ of the release. Moreover, the Weibull model was shown to be the best fitting model for free ATO and for CUR entrapped within the NLCs with solid:liquid lipids ratios of 50:50 and 75:25. In the case of the empirical Weibull model, the release profiles are characterized through the curve shape based on the $c_{3}$ parameter, defined as the "shape parameter". The $c_{3}$ values, in which $c_{3}>1$ for free ATO and $c_{3}<1$ for CUR in 50:50 and 75:25 NLCs, suggest that the release profiles display an S-shaped with upward curvature followed by a turning point, and an exponential shape with a steep initial slope, respectively (Cascone, 2017; Dash et al., 2010). However, a lack of fitting was obtained considering all models, for free CUR, due to the CUR degradation at $\mathrm{pH}=7.4$. Fig. 20 presents the release profiles estimated using the best fitting model for each formulation.

\subsection{Biomimeting usNLC}

usNLCs were coated with RBCs membranes aiming at enhancing the pharmacokinetic characteristics of the nanosystems. In fact, cell membrane-coating technology may promote a stealth behavior and reduce macrophage recognition and in vivo elimination, being as such considered a promising strategy against glioblastoma (Basso et al., 2020). Following a co-extrusion process, the volume of RBC vesicles, the number of co-extrusions, as well as the concentration of stearylamine (cationic compound) were considered as potentially critical parameters affecting the quality of the nanosystems, in particular particle size, polydispersity index, and zeta potential (Chai et al., 2017; Luk et al., 2014).

The Taguchi $\mathrm{L}_{9}, 3^{3}$ orthogonal array design was used to optimize the coating procedure. The number of co-extrusions ( 3,9 and 15), the volume of RBC vesicles $(50,100$ and $150 \mu \mathrm{L}$ ) and the concentration of stearylamine (0, 1 and $2 \mathrm{mM}$ ), reflecting different surface charges of the lipid core, were selected as independent factors and their effect on formulations parameters was assessed, resulting in a total of 9 formulations. The $\mathrm{S} / \mathrm{N}$ ratio was calculated to determine significance of 

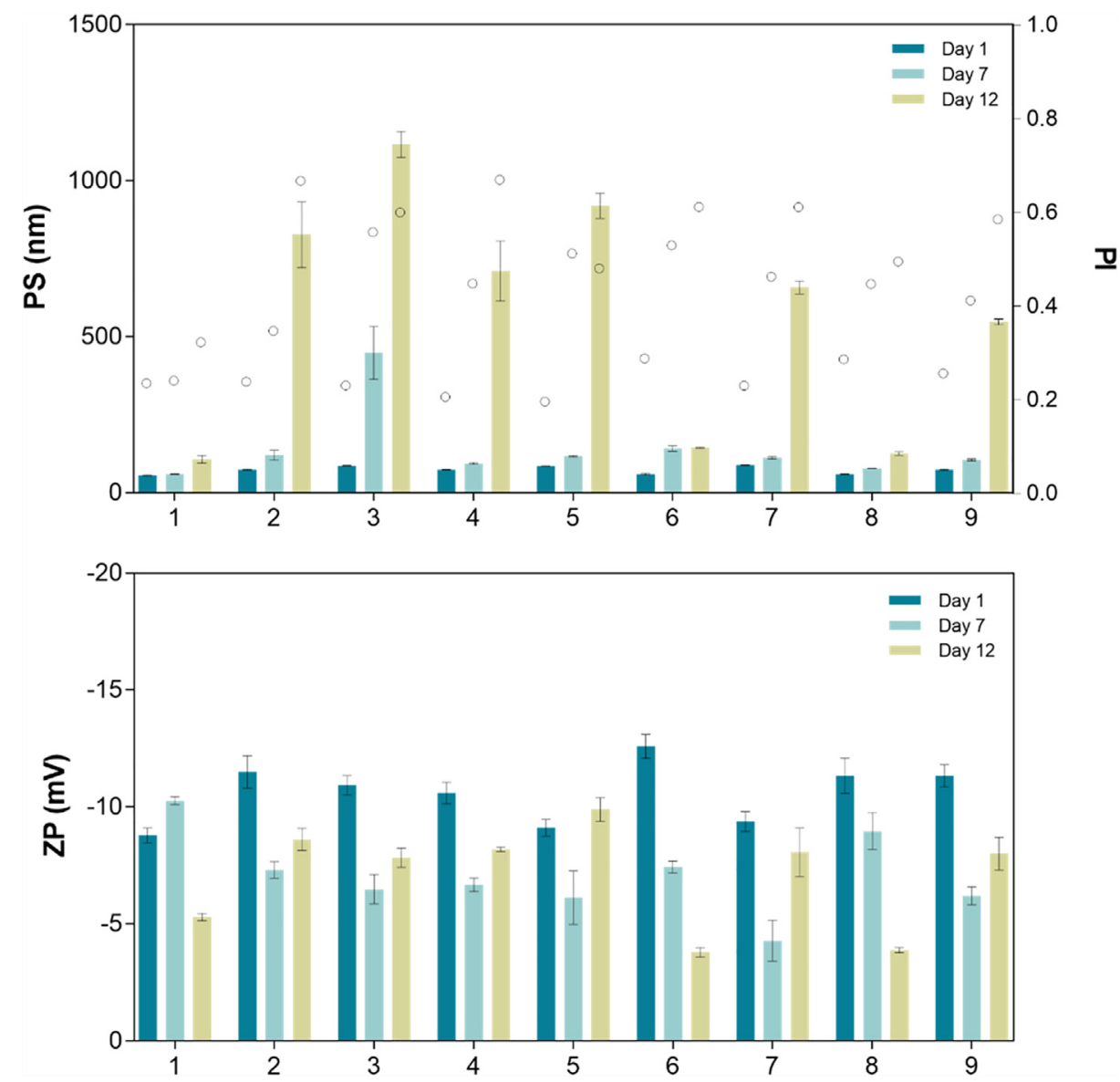

Fig. 21. Monitoring of the physicochemical stability of biomimeting usNLCs through the evaluation of PS, PI and ZP at 1, 7 and 12 days after production.

Table 9

Exploratory Taguchi design matrix and experimental and predicted mean PS and S/N ratios.

\begin{tabular}{|c|c|c|c|c|c|c|c|}
\hline \multirow[t]{2}{*}{ Exp. } & \multicolumn{3}{|l|}{ Factor Levels } & \multicolumn{2}{|l|}{ Mean PS (nm) } & \multicolumn{2}{|l|}{$\mathrm{S} / \mathrm{N}$ ratio } \\
\hline & Number of Co-extrusions & Volume of RBC vesicles & Stearylamine & Experimental & Predicted & Experimental & Predicted \\
\hline 1 & -1 & -1 & -1 & 55.14 & 57.02 & -34.83 & -35.16 \\
\hline 2 & -1 & 0 & 0 & 73.56 & 71.79 & -37.33 & -36.99 \\
\hline 3 & -1 & 1 & 1 & 86.51 & 86.57 & -38.74 & -38.82 \\
\hline 4 & 0 & -1 & 0 & 73.63 & 72.26 & -37.34 & -37.03 \\
\hline 5 & 0 & 0 & 1 & 85.10 & 87.03 & -38.60 & -38.86 \\
\hline 6 & 0 & 1 & -1 & 59.62 & 58.72 & -35.51 & -35.44 \\
\hline 7 & 1 & -1 & 1 & 88.22 & 87.49 & -38.91 & -38.90 \\
\hline 8 & 1 & 0 & -1 & 58.90 & 59.18 & -35.40 & -35.48 \\
\hline 9 & 1 & 1 & 0 & 73.32 & 73.95 & -37.31 & -37.31 \\
\hline
\end{tabular}

each independent factor and their optimum levels for the optimized formulation. The obtained results for PS, PI and ZP of the experiments are displayed in Fig. 21, as well as their stability at 1, 7 and 12 days after production. Formulations without stearylamine, i.e, with a lipid core surface charge of $-17 \mathrm{mV}$ (usNLCs containing a solid:liquid lipid ratio of 25:75), were the most stable along 12 days, showing an acceptable stability behavior. Overall, at 12 days, the remaining formulations displayed a significant increase in PS, PI and ZP. Since the variation of PI and ZP values were not statistically significant, PS was evaluated as the key dependent factor. The $\mathrm{S} / \mathrm{N}$ response ratios are shown in Table 9. The main effects of each factor are depicted in Fig. 22, which represents each level as $\mathrm{x}$-axis and mean PS, or S/N ratio as $y$-axis. The presence of stearylamine and consequently the lipid core surface charge is the most impacting factor on PS, expressing a larger S/ $\mathrm{N}$ ratio range. An increase in surface charge to less negative values leads to the production of larger particles with lower stability, as indicated by the lower $\mathrm{S} / \mathrm{N}$ ratios. Moreover, no significant variations were found for the $\mathrm{S} / \mathrm{N}$ ratios corresponding to the other factors, specifically, for the number of co-extrusions and the volume of RBC vesicles.

The predictive PS achieved through the linear regression defined in Eq. (7) and the respective $\mathrm{S} / \mathrm{N}$ ratios are represented in Table 9. Note that the $\mathrm{L}_{9}, 3^{3}$ orthogonal design showed a great predictability for all the experimented combinations.

PS $(\mathrm{nm})=72.67+0.87 \times$ Number of co-extrusions

$$
\begin{aligned}
& +0.41 \times \text { Volume of } R B C \text { vesicles } \\
& +14.36 \times \text { Concentration of Stearylamine }
\end{aligned}
$$

Considering the highest $\mathrm{S} / \mathrm{N}$ ratios obtained for PS, the optimal level combination corresponds to the usNLCs with a 25:75 solid:liquid lipid ratio, to which $50 \mu \mathrm{L}$ of RBC vesicles were added and extruded through a $100 \mathrm{~nm}$ polycarbonate membrane 3 times. These conditions are 


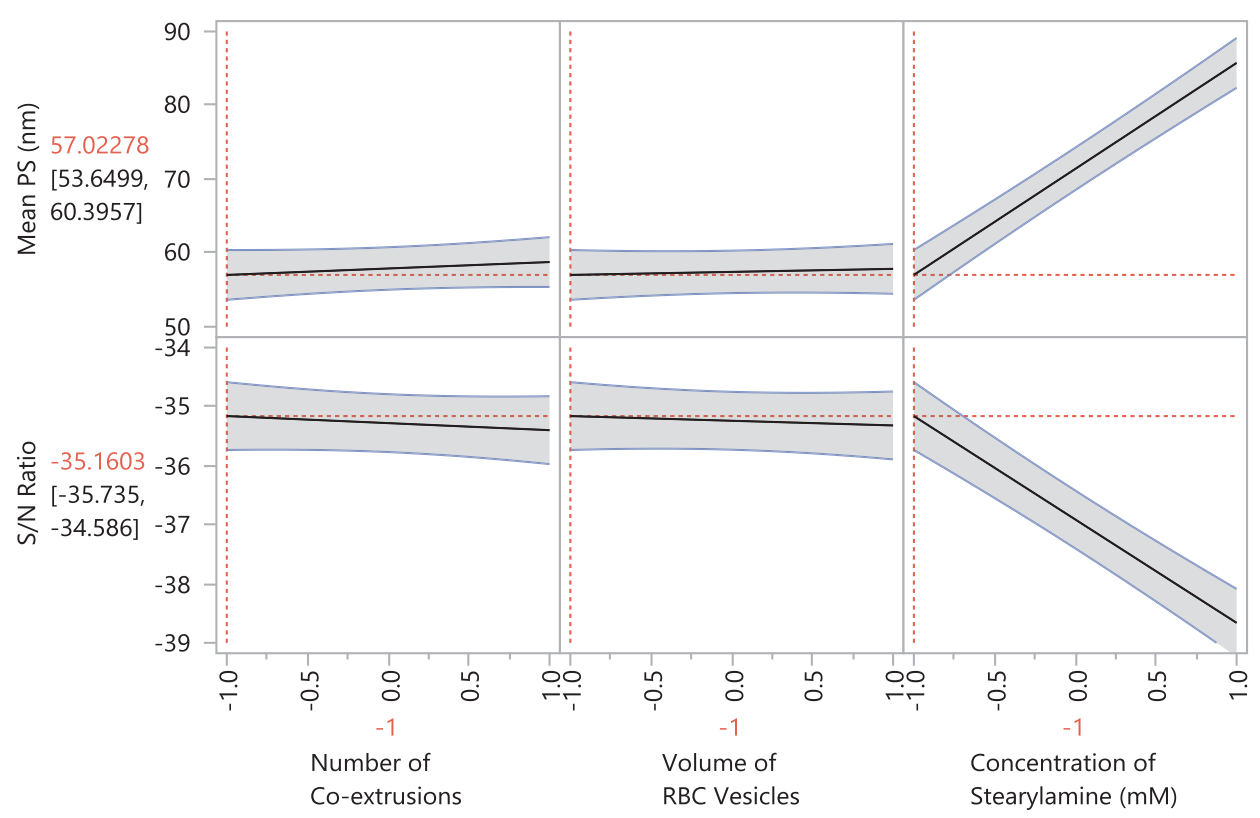

Fig. 22. Taguchi main effects plot for mean PS and S/N ratios considering the three levels of each factor. Grey darker areas represent $95 \%$ confidence intervals for mean PS and S/N Ratio.

elicited to provide smaller and stable biomimeting usNLCs and have been taken into consideration for further performance studies.

\subsection{Overview}

A crosswise analysis of the DoE outcomes points out to the following assumptions:

(i) From $3^{2}$ full factorial design, nanoparticle template composition is settled on a low solid:liquid ratio and high surfactant concentration. This formulation meets the desired quality target profile, including a small particle size and narrow size distribution, along with maximized drug loading properties and stability.

(ii) From $2^{2}$ full factorial design, method interchangeability is validated, which opens avenues to up-scaling manufacturing.

(iii) From Taguchi design, biomimeting ultra-small lipid nanoconstructs were retrieved, leading the way to surrogate strategies in glioblastoma treatment.

\section{Conclusions}

Understanding the BBB crossability at a molecular level is fundamental for drug repurposing in glioblastoma. In this work, a computational framework based on MD simulations proved useful to screen the co-delivery of atorvastatin calcium and curcumin.

QbD principles, with focus on risk assessment, were set forth throughout the development of the formulations, with the attributes and process parameters of higher risk being the solid:liquid lipid ratio, type and concentration of liquid lipids and surfactants, along with the type of production method.

The optimization studies based on multivariate analysis and design of experiments led to the production of usNLCs with the desired predefined characteristics, which were supported on (i) low concentrations of solid lipid, (ii) high concentrations of the aqueous surfactant Tween ${ }^{\circledast}$ 80 and, (iii) both ultrasonication and high pressure homogenization methods. usNLCs exhibited a sustained co-release pattern of the compounds and stability up to 6 months.

Finally, usNLCs were successfully coated with erythrocyte membranes, as a strategy to fit glioblastoma passive targeting through the enhanced permeability and retention effect. Further in vitro/in vivo studies are under way to establish the proof-of-concept of this biomodel drug delivery system.

\section{CRediT authorship contribution statement}

Maria Mendes: Writing - original draft, Investigation, Data curation. Joào Basso: Writing - original draft, Investigation, Data curation. Jessica Silva: Writing - original draft, Investigation, Data curation. Tânia Cova: Formal analysis, Writing - review \& editing. Joảo Sousa: Methodology, Funding acquisition, Resources, Project administration, Supervision. Alberto Pais: Formal analysis, Writing - review \& editing, Methodology, Funding acquisition, Resources, Project administration, Supervision. Carla Vitorino: Writing - original draft, Formal analysis, Writing - review \& editing, Methodology, Funding acquisition, Resources, Project administration, Supervision.

\section{Declaration of Competing Interest}

The authors declare that they have no known competing financial interests or personal relationships that could have appeared to influence the work reported in this paper.

\section{Acknowledgements}

The authors acknowledge the Portuguese Agency for Scientific Research, "Fundação para a Ciência e a Tecnologia (FCT), the Portuguese Agency for Scientific Research", for the financial support through the projects POCI-01-0145-FEDER-016648, PEst-UID/NEU/ 04539/2013 and COMPETE (Ref. POCI-01-0145-FEDER-007440). The Coimbra Chemistry Centre (CQC) is also supported by FCT through the Project UID/QUI/00313/2020. Maria Mendes and João Basso acknowledge the $\mathrm{PhD}$ research Grants SFRH/BD/133996/2017 and SFRH/BD/149138/2019, respectively, assigned by FCT.

\section{Appendix A. Supplementary data}

Supplementary data to this article can be found online at https:// doi.org/10.1016/j.ijpharm.2020.119661. 


\section{References}

Alifieris, C., Trafalis, D.T., 2015. Glioblastoma multiforme: Pathogenesis and treatment. Pharmacol. Ther. 152, 63-82. https://doi.org/10.1016/j.pharmthera.2015.05.005.

Abdi, H., Williams, L.J., 2010. Principal component analysis, Wiley Interdiscip. Rev. Comput. Stat. 2, 433-459. https://doi.org/10.1002/wics.101.

Abdi, H., 2010. Partial least squares regression and projection on latent structure regression (PLS Regression). Wiley Interdiscip. Rev. Comput. Stat. 2, 97-106. https:// doi.org/10.1002/wics.51.

Basso, J., Mendes, M., Cova, T.F.G.G., Sousa, J.J., Pais, A.A.C.C., Vitorino, C., 2018. Analytical Quality by Design (AQbD) as a multiaddressable platform for co-encapsulating drug assays. Anal. Methods. 10, 5659-5671. https://doi.org/10.1039/ C8AY01695J.

Berger, O., Edholm, O., Jähnig, F., 1997. Molecular dynamics simulations of a fluid bilayer of dipalmitoylphosphatidylcholine at full hydration, constant pressure, and constant temperature. Biophys. J. 72, 2002-2013. https://doi.org/10.1016/S00063495(97)78845-3.

Blasi, P., Giovagnoli, S., Schoubben, A., Puglia, C., Bonina, F., Rossi, C., Ricci, M., 2011. Lipid nanoparticles for brain targeting I. Formulation optimization. Int. J. Pharm. 419, 287-295. https://doi.org/10.1016/J.IJPHARM.2011.07.035.

Basso, J., Miranda, A., Sousa, J., Pais, A., Vitorino, C., 2018. Repurposing drugs for glioblastoma: From bench to bedside. Cancer Lett. 428, 173-183. https://doi.org/10. 1016/j.canlet.2018.04.039.

Basso, J., Silva, J., Mendes, M., Sousa, J., Pais, A., Vitorino, C., 2020. Cell MembraneMimetic Nanoparticles for Cancer Treatment: What Has Been Done So Far?, in: Nanoparticle Drug Delivery Systems for Cancer Treatment. Jenny Stanford Publishinghttps://doi.org/10.1201/9780429341250-4.

Cascone, S., 2017. Modeling and comparison of release profiles: Effect of the dissolution method. Eur. J. Pharm. Sci. 106, 352-361. https://doi.org/10.1016/j.ejps.2017.06. 021.

Carlsson, S.K., Brothers, S.P., Wahlestedt, C., 2014. Emerging treatment strategies for glioblastoma multiforme. EMBO Mol. Med. 6, 1359-1370. https://doi.org/10.15252/ emmm.201302627.

Chai, Z., Hu, X., Wei, X., Zhan, C., Lu, L., Jiang, K., Su, B., Ruan, H., Ran, D., Fang, R.H., Zhang, L., Lu, W., 2017. A facile approach to functionalizing cell membrane-coated nanoparticles with neurotoxin-derived peptide for brain-targeted drug delivery. J. Control. Release. 264, 102-111. https://doi.org/10.1016/j.jconrel.2017.08.027.

EMA, 2010. Committee for medicinal products for human use - Guideline on the Investigation of Bioequivalence.

Dejaegher, B., Vander Heyden, Y., 2011. Experimental designs and their recent advances in set-up, data interpretation, and analytical applications. J. Pharm. Biomed. Anal. 56, 141-158. https://doi.org/10.1016/j.jpba.2011.04.023.

Davis, R., John, P., 2018. Application of Taguchi-Based Design of Experiments for Industrial Chemical Processes, in: Statistical Approaches with Emphasis on Design of Experiments Applied to Chemical Processes. Intechopen 137. https://doi.org/10. 5772/intechopen.69501.

Dash, S., Murthy, P.N., Nath, L., Chowdhury, P., 2010. Kinetic modeling on drug release from controlled drug delivery systems. Acta Pol. Pharm. 67, 217-223.

Elmowafy, M., Ibrahim, H.M., Ahmed, M.A., Shalaby, K., Salama, A., Hefesha, H., 2017. Atorvastatin-loaded nanostructured lipid carriers (NLCs): strategy to overcome ora delivery drawbacks. Drug Deliv. 24, 932-941. https://doi.org/10.1080/10717544. 2017.1337823.

Gelperina, S., Maksimenko, O., Khalansky, A., Vanchugova, L., Shipulo, E., Abbasova, K., Berdiev, R., Wohlfart, S., Chepurnova, N., Kreuter, J., 2010. Drug delivery to the brain using surfactant-coated poly(lactide-co-glycolide) nanoparticles: Influence of the formulation parameters. Eur. J. Pharm. Biopharm. 74, 157-163. https://doi.org/ 10.1016/J.EJPB.2009.09.003.

Göppert, T.M., Müller, R.H., 2005. Polysorbate-stabilized solid lipid nanoparticles as colloidal carriers for intravenous targeting of drugs to the brain: Comparison of plasma protein adsorption patterns. J. Drug Target. 13, 179-187. https://doi.org/10. 1080/10611860500071292.

Gupta, S., Kesarla, R., Chotai, N., Misra, A., Omri, A., 2017. Systematic approach for the formulation and optimization of solid lipid nanoparticles of efavirenz by high pressure homogenization using design of experiments for brain targeting and enhanced bioavailability. Biomed Res. Int. 2017, 5984014. https://doi.org/10.1155/2017/ 5984014.

Ganesan, P., Narayanasamy, D., 2017. Lipid nanoparticles: Different preparation techniques, characterization, hurdles, and strategies for the production of solid lipid nanoparticles and nanostructured lipid carriers for oral drug delivery. Sustain. Chem. Pharm. 6, 37-56. https://doi.org/10.1016/J.SCP.2017.07.002.

Gersey, Z.C., Rodriguez, G.A., Barbarite, E., Sanchez, A., Walters, W.M., Ohaeto, K.C., Komotar, R.J., Graham, R.M., 2017. Curcumin decreases malignant characteristics of glioblastoma stem cells via induction of reactive oxygen species. BMC Cancer. 17, 99. https://doi.org/10.1186/s12885-017-3058-2.

Hu, C.-M.J., Zhang, L., Aryal, S., Cheung, C., Fang, R.H., Zhang, L., 2011. Erythrocyte membrane-camouflaged polymeric nanoparticles as a biomimetic delivery platform. Proc. Natl. Acad. Sci. 108, 10980-10985.

Humphrey, W., Dalke, A., Schulten, K., 1996. VMD: visual molecular dynamics. J. Mol. Graph. 14, 33-38.

Hess, B., Bekker, H., Berendsen, H.J.C., Fraaije, J.G.E.M., 1997. LINCS: a linear constraint solver for molecular simulations. J. Comput. Chem. 18, 1463-1472.

Hess, B., Kutzner, C., van der Spoel, D., Lindahl, E., 2008. GROMACS 4: algorithms for highly efficient, load-balanced, and scalable molecular simulation. J. Chem. Theory Comput. 4, 435-447. https://doi.org/10.1021/ct700301q.

G. Iacob, E.B. Dinca, Current data and strategy in glioblastoma multiforme., J. Med. Life.
2 (n.d.) $386-93$.

I.C. on Harmonisation, ICH Harmonised Tripartite Guideline - Quality Risk Management Q9, 2005.

Jolliffe, I.T., 2002. Principal Component Analysis, 2 nd, Springer-Verlag. New York. https://doi.org/10.1007/b98835.

Jones, H.M., Fang, Z., Sun, W., Clark, L.H., Stine, J.E., Tran, A.Q., Sullivan, S.A., Gilliam, T.P., Zhou, C., Bae-Jump, V.L., 2017. Atorvastatin exhibits anti-tumorigenic and antimetastatic effects in ovarian cancer in vitro. Am. J. Cancer Res. 7, 2478.

Karim, R., Palazzo, C., Evrard, B., Piel, G., 2016. Nanocarriers for the treatment of glioblastoma multiforme: Current state-of-the-art. J. Control. Release. 227, 23-37. https://doi.org/10.1016/j.jconrel.2016.02.026.

Khan, S., Baboota, S., Ali, J., Khan, S., Narang, R., Narang, J., 2015. Nanostructured lipid carriers: An emerging platform for improving oral bioavailability of lipophilic drugs. Int. J. Pharm. Investig. 5, 182. https://doi.org/10.4103/2230-973X.167661.

Kassem, A.M., Ibrahim, H.M., Samy, A.M., 2017. Development and optimisation of atorvastatin calcium loaded self-nanoemulsifying drug delivery system (SNEDDS) for enhancing oral bioavailability: in vitro and in vivo evaluation. J. Microencapsul. 34, 319-333. https://doi.org/10.1080/02652048.2017.1328464.

Kaur, I.P., Bhandari, R., Bhandari, S., Kakkar, V., 2008. Potential of solid lipid nanoparticles in brain targeting. J. Control. Release. 127, 97-109. https://doi.org/10. 1016/J.JCONREL.2007.12.018.

Luthra, P.M., Lal, N., 2016. Prospective of curcumin, a pleiotropic signalling molecule from Curcuma longa in the treatment of Glioblastoma. Eur. J. Med. Chem. 109, 23-35. https://doi.org/10.1016/j.ejmech.2015.11.049.

Leong S.W., Bioinformatics for Membrane Lipid Simulations: Models, Computational Methods, and Web Server Tools, in: T.S. Lim (Ed.), IntechOpen, Rijeka, 2016: p. Ch. 4. doi:10.5772/62576.

Lasoń, E., Sikora, E., Ogonowski, J., 2013. Influence of process parameters on properties of Nanostructured Lipid Carriers (NLC) formulation. Acta Biochim. Pol. 60, 773-777.

Luk, B.T., Hu, C.M.J., Fang, R.H., Dehaini, D., Carpenter, C., Gao, W., Zhang, L., 2014. Interfacial interactions between natural RBC membranes and synthetic polymeric nanoparticles. Nanoscale. 6, 2730-2737.

Lee, S.Y., 2016. Temozolomide resistance in glioblastoma multiforme. Genes Dis. 3, 198-210. https://doi.org/10.1016/j.gendis.2016.04.007.

Mendes, M., Soares, H.T., Arnaut, L.G., Sousa, J.J., Pais, A., Vitorino, C., 2016. Can lipid nanoparticles improve intestinal absorption? Int. J. Pharm. 515, 69-83. https://doi. org/10.1016/j.ijpharm.2016.09.065.

Mendes, M., Miranda, A., Cova, T., Gonçalves, L., Almeida, A.J., Sousa, J.J., do Vale, M.L.C., Marques, E.F., Pais, A., Vitorino, C., 2018. Modeling of ultra-small lipid nanoparticle surface charge for targeting glioblastoma. European Journal of Pharmaceutical Sciences 117, 255-269. https://doi.org/10.1016/j.ejps.2018.02.024.

Mutihac, L., Mutihac, R., 2008. Mining in chemometrics. Anal. Chim. Acta. 612, 1-18. https://doi.org/10.1016/J.ACA.2008.02.025.

Malde, A.K., Zuo, L., Breeze, M., Stroet, M., Poger, D., Nair, P.C., Oostenbrink, C., Mark, A.E., 2011. An Automated Force Field Topology Builder (ATB) and Repository: Version 1.0. J. Chem. Theory Comput. 7, 4026-4037. https://doi.org/10.1021/ ct200196m.

Miranda, A., Cova, T., Sousa, J., Vitorino, C., Pais, A., 2018. Computational modeling in glioblastoma: from the prediction of blood-brain barrier permeability to the simulation of tumor behavior. Future Med. Chem. 10, 121-131.

Mendes, M., Miranda, A., Cova, T., Gonçalves, L., Almeida, A.J., Sousa, J.J., do Vale Marques, E.F., Pais, A., Vitorino, C., 2018. Modeling of ultra-small lipid nanoparticle surface charge for targeting glioblastoma. Eur. J. Pharm. Sci. 117, 255-269. https:// doi.org/10.1016/j.ejps.2018.02.024.

Miranda, A., Blanco-Prieto, M.J., Sousa, J., Pais, A., Vitorino, C., 2017. Breaching barriers in glioblastoma. Part II: Targeted drug delivery and lipid nanoparticles. Int. J. Pharm. 531, 389-410. https://doi.org/10.1016/j.ijpharm.2017.07.049.

Miranda, A., Blanco-Prieto, M., Sousa, J., Pais, A., Vitorino, C., 2017. Breaching barriers in glioblastoma. Part I: Molecular pathways and novel treatment approaches. Int. J. Pharm. 531, 372-388. https://doi.org/10.1016/j.ijpharm.2017.07.056.

Neves, A.R., Queiroz, J.F., Weksler, B., Romero, I.A., Couraud, P.O., Reis, S., 2015. Solid lipid nanoparticles as a vehicle for brain-targeted drug delivery: two new strategies of functionalization with apolipoprotein E. Nanotechnology 26, 495103. https://doi. org/10.1088/0957-4484/26/49/495103.

Ohgaki, H., Kleihues, P., 2005. Population-Based Studies on Incidence, Survival Rates, and Genetic Alterations in Astrocytic and Oligodendroglial Gliomas. J. Neuropathol Exp. Neurol. 64, 479-489. https://doi.org/10.1093/jnen/64.6.479.

Perrault, S.D., Walkey, C., Jennings, T., Fischer, H.C., Chan, W.C.W., 2009. Mediating tumor targeting efficiency of nanoparticles through design. Nano Lett. 9, 1909-1915. https://doi.org/10.1021/nl900031y.

Piggot, T.J., Piñeiro, Á., Khalid, S., 2012. Molecular dynamics simulations of phosphatidylcholine membranes: a comparative force field study. J. Chem. Theory Comput. 8, 4593-4609. https://doi.org/10.1021/ct3003157.

Prabhu, P., Patravale, V., 2016. Dissolution enhancement of atorvastatin calcium by cogrinding technique. Drug Deliv. Transl. Res. 6, 380-391. https://doi.org/10.1007/ s13346-015-0271-x.

Pourgholi, F., Hajivalili, M., Farhad, J.N., Kafil, H.S., Yousefi, M., 2016. Nanoparticles: Novel vehicles in treatment of Glioblastoma. Biomed. Pharmacother. 77, 98-107. https://doi.org/10.1016/j.biopha.2015.12.014.

Shamloo, A., Pedram, M.Z., Heidari, H., Alasty, A., 2016. Computing the blood brain barrier (BBB) diffusion coefficient: A molecular dynamics approach. J. Magn. Magn. Mater. 410, 187-197. https://doi.org/10.1016/J.JMMM.2016.03.030.

Silva, J., Mendes, M., Cova, T., Sousa, J., Pais, A., Vitorino, C., 2018. Unstructured formulation data analysis for the optimization of lipid nanoparticle drug delivery vehicles. AAPS Pharm. Sci. Tech. 1-12. https://doi.org/10.1208/s12249-018-1078-0.

Singh, R., Lillard, J.W., 2009. Nanoparticle-based targeted drug delivery. Exp. Mol. 
Pathol. 86, 215-223. https://doi.org/10.1016/j.yexmp.2008.12.004.

Stupp, R., Mason, W.P., Van Den Bent, M.J., Weller, M., Fisher, B., Taphoorn, M.J.B., Belanger, K., Brandes, A.A., Marosi, C., Bogdahn, U., 2005. Radiotherapy plus concomitant and adjuvant temozolomide for glioblastoma. N. Engl. J. Med. 352, 987-996.

Sordillo, L.A., Sordillo, P.P., Helson, L., 2015. Curcumin for the Treatment of Glioblastoma. Anticancer Res. 35, 6373-6378.

Tapeinos, C., Battaglini, M., Ciofani, G., 2017. Advances in the design of solid lipid nanoparticles and nanostructured lipid carriers for targeting brain diseases. J. Control. Release. 264, 306-332. https://doi.org/10.1016/j.jconrel.2017.08.033.

Urbańska, K., Sokołowska, J., Szmidt, M., Sysa, P., 2014. Glioblastoma multiforme - an overview. Contemp. Oncol. 18, 307-312. https://doi.org/10.5114/wo.2014.40559.

University of Calgary, Biocomputing Group, Using Comput. Simulations to Study Biol. Probl. (n.d.).

Vasilev, A., Sofi, R., Tong, L., Teschemacher, A., Kasparov, S., 2018. In Search of a
Breakthrough Therapy for Glioblastoma Multiforme. Neuroglia 1, 292-310. https:// doi.org/10.3390/neuroglia1020020.

Vitorino, C., Almeida, J., Gonçalves, L.M., Almeida, A.J., Sousa, J.J., Pais, A.A.C.C., 2013 Co-encapsulating nanostructured lipid carriers for transdermal application: From experimental design to the molecular detail. J. Control. Release. 167, 301-314. https://doi.org/10.1016/j.jconrel.2013.02.011.

Varmuza, K., Filzmoser, P., 2009. Introduction to multivariate statistical analysis in chemometrics. CRC Press.

Yin, H., Zhou, Y., Wen, C., Zhou, C., Zhang, W., Hu, X., Wang, L., You, C., Shao, J., 2014 Curcumin sensitizes glioblastoma to temozolomide by simultaneously generating ROS and disrupting AKT/mTOR signaling. Oncol. Rep. 32, 1610-1616. https://doi. org/10.3892/or.2014.3342.

Zhuang, W., Long, L., Zheng, B., Ji, W., Yang, N., Zhang, Q., Liang, Z., 2012. Curcumin promotes differentiation of glioma-initiating cells by inducing autophagy. Cancer Sci. 103, 684-690. https://doi.org/10.1111/j.1349-7006.2011.02198.x. 